\title{
Search for the Higgs Boson Decaying to Two Muons in Proton-Proton Collisions at $\sqrt{s}=13 \mathrm{TeV}$
}

\author{
A. M. Sirunyan et al. \\ (CMS Collaboration)
}

(Received 17 July 2018; revised manuscript received 28 October 2018; published 14 January 2019)

\begin{abstract}
A search for the Higgs boson decaying to two oppositely charged muons is presented using data recorded by the CMS experiment at the CERN LHC in 2016 at a center-of-mass energy $\sqrt{s}=13 \mathrm{TeV}$, corresponding to an integrated luminosity of $35.9 \mathrm{fb}^{-1}$. Data are found to be compatible with the predicted background. For a Higgs boson with a mass of $125.09 \mathrm{GeV}$, the $95 \%$ confidence level observed (background-only expected) upper limit on the production cross section times the branching fraction to a pair of muons is found to be 3.0 (2.5) times the standard model expectation. In combination with data recorded at center-of-mass energies $\sqrt{s}=7$ and $8 \mathrm{TeV}$, the background-only expected upper limit improves to 2.2 times the standard model value with a standard model expected significance of 1.0 standard deviation. The corresponding observed upper limit is 2.9 with an observed significance of 0.9 standard deviation. This corresponds to an observed upper limit on the standard model Higgs boson branching fraction to muons of $6.4 \times 10^{-4}$ and to an observed signal strength of $1.0 \pm 1.0$ (stat) \pm 0.1 (syst).
\end{abstract}

DOI: 10.1103/PhysRevLett.122.021801

In the standard model (SM), the masses of fermions are generated by their Yukawa coupling to the Higgs field [1-4], whose existence was confirmed by the Higgs boson $(H)$ discovery [5-7]. Measurements at CMS and ATLAS provided evidence that the Higgs boson couples to bottom quarks $[8,9]$ and established that it couples with tau leptons $[10,11]$ and top quarks $[12,13]$. The Higgs boson mass has been measured and is found to be $m_{\mathrm{H}}=125.09 \pm$ $0.24 \mathrm{GeV}$ in a combination of ATLAS and CMS data samples [14]. The study of the Higgs boson decays to muons is of particular importance, because it extends the investigation to its couplings to fermions of the second generation. For a Higgs boson with a mass of $125.09 \mathrm{GeV}$, the expected branching fraction $(\mathcal{B})$ to muons is $2.17 \times 10^{-4}[15]$, and the narrow decay width of the Higgs boson $[16,17]$ is several orders of magnitude smaller than the $O(\mathrm{GeV})$ experimental dimuon mass resolution. The signal would appear as a narrow resonance over a smoothly falling mass spectrum from the SM background processes, primarily Drell-Yan (DY) and leptonic $t \bar{t}$ decays.

The CMS and ATLAS Collaborations placed upper limits on the product of the Higgs boson production cross section and branching fraction $\mathcal{B}\left(H \rightarrow \mu^{+} \mu^{-}\right)$of approximately 7 times the SM value at $95 \%$ confidence level

\footnotetext{
*Full author list given at the end of the article.

Published by the American Physical Society under the terms of the Creative Commons Attribution 4.0 International license. Further distribution of this work must maintain attribution to the author(s) and the published article's title, journal citation, and DOI. Funded by SCOAP ${ }^{3}$.
}

(C.L.) with LHC run 1 data $[18,19]$, collected at centerof-mass energies $\sqrt{s}=7$ and $8 \mathrm{TeV}$. The ATLAS Collaboration improved its expected limit to 2.9 times the SM expectation by adding $36.1 \mathrm{fb}^{-1}$ of data collected at $13 \mathrm{TeV}$ [20] and measured an observed limit of 2.8 times the SM expectation. This Letter presents a search for $H \rightarrow \mu^{+} \mu^{-}$events with the CMS detector using $35.9 \mathrm{fb}^{-1}$ of proton-proton ( $p p$ ) collision data collected in 2016 at $\sqrt{s}=13 \mathrm{TeV}$ and its combination with the data collected at $\sqrt{s}=7$ and $8 \mathrm{TeV}$ corresponding to integrated luminosities of 5.0 and $19.7 \mathrm{fb}^{-1}$, respectively.

The central feature of the CMS apparatus is a superconducting solenoid of $6 \mathrm{~m}$ internal diameter, providing a magnetic field of $3.8 \mathrm{~T}$. Within the solenoid volume are a silicon pixel and strip tracker, a lead tungstate crystal electromagnetic calorimeter, and a brass and scintillator hadron calorimeter, each composed of a barrel and two end cap sections. Forward calorimeters extend the pseudorapidity $(\eta)$ coverage provided by the barrel and end cap detectors. Muons are detected in gas-ionization detectors embedded in the steel flux-return yoke outside the solenoid. A more detailed description of the CMS detector, together with a definition of the coordinate system used and the relevant kinematic variables, can be found in Ref. [21].

The Monte Carlo (MC) simulated events used to model the signal include the four leading Higgs boson production processes: gluon-gluon fusion $(g g H)$, vector boson fusion (VBF), and associated production with a vector boson $(\mathrm{V} H, \mathrm{~V}=W$ or $Z$ ) or top quarks $(t \bar{t} H)$. The Higgs boson MC samples are generated at next-to-leading order (NLO) for masses of 120,125 , and $130 \mathrm{GeV}$ with POWHEG2.0 [22], 
using the parton distribution function sets of NNPDF3.0 [23]. The $g g H$ acceptance in each analysis category is found to be in agreement with that calculated at NLO with the MADGRAPH5_aMc@NLO2.2.2 [24] generator. The SM background processes considered are DY, single and pair production of top quarks ( $s t$ and $t \bar{t}$, respectively), and diand triboson production ( $\mathrm{VV}$ and $\mathrm{VVV}$, respectively). Simulated background processes are used only to optimize the event selection and not for the final background estimate, which is obtained from the data. Background samples are generated using MADGRAPH5_aMC@NLO and POWHEG. Spin correlations in multiboson processes generated using MADGRAPH 5_aMC@NLO are simulated using MADSPIN [25]. The parton shower and hadronization processes are modeled by the PYTHIA8.212 [26] generator with the CUETP8M1 [27] underlying event tune. The detector response is based on a detailed description of the CMS detector and is simulated with the GEANT4 package [28]. Simultaneous $p p$ interactions overlapping the event of interest (pileup) are included in the simulated samples. The distribution of the number of additional interactions per bunch crossing in the simulation corresponds to that observed in the $13 \mathrm{TeV}$ data collected in 2016, with an average of 23 interactions. The SM Higgs boson cross section and branching fractions are taken from the LHC Higgs boson cross section working group recommendations [15], while cross sections for the background processes are taken from FEWZ3.1 [29], TOP++2.0 [30], HATHOR [31,32], and MCFM [33].

The particle-flow (PF) algorithm [34] is used to reconstruct observable particles in each event. It combines all subdetector information to reconstruct individual particles and identify them as charged or neutral hadrons, photons, or leptons. Electron and muon candidates are formed by associating a track in the silicon detectors with a cluster of energy in the electromagnetic calorimeter [35] or a track in the muon system. The relative transverse momentum $\left(p_{T}\right)$ resolution of muon candidates with $p_{T}<100 \mathrm{GeV}$ is $\lesssim 1.6 \%$ in the barrel [36]. Jets are reconstructed using the anti- $k_{T}$ clustering algorithm [37] with a distance parameter of 0.4 , as implemented in the FASTJET package [38]. Jets are required to have a minimum $p_{T}$ of $30 \mathrm{GeV}$ and a maximum $|\eta|$ of 4.7. Further identification criteria are applied in order to reject jets from pileup or noise present in the detector [39]. For jets with $|\eta|<2.4$, multivariate algorithms discriminate jets arising from the hadronization of $b$ quarks [40]. The missing transverse momentum $p_{T}^{\text {miss }}$ is defined as the magnitude of the negative vector $\vec{p}_{T}$ sum of all reconstructed particles (charged and neutral) in the event and is modified by corrections to the energy scale of reconstructed jets. The reconstructed vertex with the largest value of summed physics object $p_{T}{ }^{2}$ is taken to be the primary $p p$ interaction vertex.

Events are selected by the trigger system requiring the presence of at least one isolated muon with $p_{T}>24 \mathrm{GeV}$
[41]. The offline selection, optimized to maximize the sensitivity of the analysis, requires at least two oppositely charged muons with $p_{T}>26 \mathrm{GeV}\left(p_{T}>20 \mathrm{GeV}\right)$ for the leading (subleading) muon and $|\eta|<2.4$. To reject events with muons from nonprompt decays, muons must be isolated, with a relative isolation sum $<25 \%$. The relative isolation sum is calculated as the scalar $p_{T}$ sum of PF objects, excluding the muon, within a cone of radius $\Delta R=\sqrt{(\Delta \eta)^{2}+(\Delta \phi)^{2}}=0.4$ centered on the direction of the muon, and divided by the muon $p_{T}$. Charged particles not associated with the event vertex are not considered in this sum, and a correction is applied in order to account for the neutral particle contamination arising from pileup [42]. The invariant mass of the Higgs boson candidate $\left(m_{\mu \mu}\right)$ is constructed from the two highest $p_{T}$ oppositely charged muons, and the event is retained for further analysis if $110<m_{\mu \mu}<150 \mathrm{GeV}$. The overall trigger efficiency for these events is $98.5 \%$.

Events are classified into categories using variables that are largely uncorrelated with $m_{\mu \mu}$ in order to enhance the sensitivity to the Higgs boson signal. The primary Higgs boson production mechanisms targeted by this analysis are $\mathrm{VBF}$ and $g g H$. The $p_{T}$ and $\eta$ of the dimuon system, and the $|\Delta \eta|$ and $|\Delta \phi|$ between the muons, distinguish between $g g H$ signal events and the DY background. The $|\eta|$ of each of the two highest $p_{T}$ jets, the mass and $|\Delta \eta|$ between the jets in each of the two highest mass dijet pairs, and the number of jets with $|\eta|<2.4$ (central jets) and $|\eta|>2.4$ (forward jets) identify VBF signal events. Finally, the number of $b$-tagged jets and $p_{T}^{\text {miss }}$ identify events with $t \bar{t}$ decays. These variables are used as input to a boosted decision tree (BDT) [43], which was trained with simulated signal and background events normalized to their respective SM cross sections. The dimuon mass and its resolution are not used as input to the BDT in order to avoid biasing the background shape but are used in the signal extraction as discussed later. Simulated signal events used in the training steps are not used later in the analysis. Figure 1 shows the BDT output distributions for data and for simulated events. The output of the classifier was transformed such that the sum of all signal events has a uniform distribution. A large fraction of the VBF signal events can be distinguished from background processes and corresponds to events with the highest BDT score.

The event categories are defined using the BDT score and the expected dimuon mass resolution, gauged by the largest $|\eta|$ of the two muons. The best mass resolution is obtained when both muons are located in the central part of the detector $|\eta|<0.9$, where the muon momentum resolution is approximately constant, and degrades when one of the muons is more forward, especially in the region $|\eta|>1.9$, where there are reduced lever arm and increased multiple scattering within the tracking volume.

The number of categories and the values of the BDT and $|\eta|$ boundaries of the categories were optimized according 


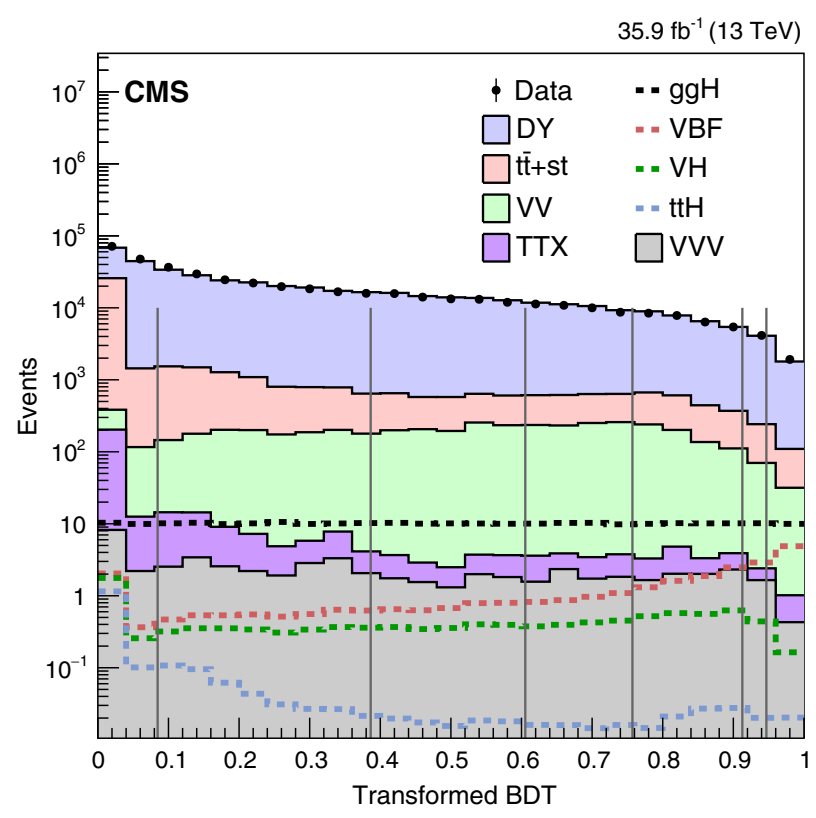

FIG. 1. The transformed BDT output distributions in data (solid points) and MC simulation (histograms). The stacked solid histograms represent the background processes, while the stacked dashed histograms represent the signal. In the legend, $\mathrm{V}$ denotes the vector bosons $W$ and $Z$, and TTX indicates the top quark pair production in association with a vector boson $\mathrm{V}$ or another top quark pair. The vertical lines denote the BDT response intervals indicated in Table I.

to an iterative process using $\sum_{i} S_{i}^{2} / B_{i}$ as a figure of merit, where $S_{i}$ and $B_{i}$ are the number of expected signal and background events, respectively, in each category in the $i$ th mass bin from 120 to $130 \mathrm{GeV}$ with $0.5 \mathrm{GeV}$ spacing. A first category boundary is created by optimizing the figure of merit against all possible boundaries in $|\eta|$ and in the BDT score separately and then choosing the one with the larger gain. The process is then repeated recursively within each of the two newly created categories to create additional category boundaries within them until a set number of categories is achieved. Some rounding of the values of the boundaries was made afterward, checking that the simplification does not significantly worsen the expected limit.

This procedure incorporates the dimuon mass resolution into the definition of the categories, optimizing the sensitivity of the analysis. This optimization results in 15 categories shown in Table I. Simulated events are used to optimize the event categories and to estimate the selection efficiency for signal events. In each category, the shape and the normalization of the dimuon mass distribution of the background contributions are obtained from a parametric fit to the data using a set of empirical functions. The product of signal acceptance and efficiency for the $H \rightarrow \mu^{+} \mu^{-}$signal varies depending on the production process. This product is shown in Table I for each category for a Higgs boson mass of $125 \mathrm{GeV}$, together with the functional form used to derive the background from the data and the $S / \sqrt{B}$ ratio within the full width at half maximum (FWHM) of the expected signal distribution.

The reconstructed invariant mass of the signal is modeled with a sum of up to three Gaussian functions, which provides a satisfactory description of the low-mass tail of the distribution, and each model is separately fit to the

TABLE I. The optimized event categories, the product of acceptance and selection efficiency in percent for the different production processes, the total expected number of SM signal events $\left(m_{H}=125 \mathrm{GeV}\right)$, the estimated number of background events per $\mathrm{GeV}$ at $125 \mathrm{GeV}$, the FWHM of the signal peak, the background functional fit form, and the $S / \sqrt{B}$ ratio within the FWHM of the expected signal distribution.

\begin{tabular}{|c|c|c|c|c|c|c|c|c|c|c|c|}
\hline $\begin{array}{l}\text { BDT response } \\
\text { quantile }(\%)\end{array}$ & $\begin{array}{l}\text { Maximum } \\
\text { muon }|\eta|\end{array}$ & $\begin{array}{l}g g H \\
(\%)\end{array}$ & $\begin{array}{l}\mathrm{VBF} \\
(\%)\end{array}$ & $\begin{array}{l}W H \\
(\%)\end{array}$ & $\begin{array}{l}\mathrm{ZH} \\
(\%)\end{array}$ & $\begin{array}{l}t \bar{t} H \\
(\%)\end{array}$ & Signal & $\begin{array}{l}\mathrm{Bkg} / \mathrm{GeV} \\
@ 125 \mathrm{GeV}\end{array}$ & $\begin{array}{c}\text { FWHM } \\
(\mathrm{GeV})\end{array}$ & $\begin{array}{l}\text { Bkg fit } \\
\text { function }\end{array}$ & $\begin{array}{c}S / \sqrt{B} @ \\
\text { FWHM }\end{array}$ \\
\hline $0-8$ & $|\eta|<2.4$ & 4.9 & 1.3 & 3.3 & 6.3 & 32 & 21.2 & $3.13 \times 10^{3}$ & 4.2 & $\mathcal{D}_{\mathrm{MBW}} B_{\operatorname{deg} 4}$ & 0.12 \\
\hline $8-39$ & $1.9<|\eta|<2.4$ & 5.6 & 1.7 & 3.9 & 3.5 & 1.3 & 22.3 & $1.34 \times 10^{3}$ & 7.2 & $\mathcal{D}_{\mathrm{MBW}} B_{\mathrm{deg} 4}$ & 0.16 \\
\hline $8-39$ & $0.9<|\eta|<1.9$ & 10 & 2.8 & 6.5 & 6.4 & 5.2 & 41.1 & $2.24 \times 10^{3}$ & 4.1 & $\mathcal{D}_{\text {MBW }} B_{\operatorname{deg} 4}$ & 0.29 \\
\hline $8-39$ & $|\eta|<0.9$ & 3.2 & 0.8 & 1.9 & 2.1 & 3.5 & 12.7 & $7.83 \times 10^{2}$ & 2.9 & $\mathcal{D}_{\mathrm{MBW}} B_{\mathrm{deg} 4}$ & 0.18 \\
\hline $39-61$ & $1.9<|\eta|<2.4$ & 2.9 & 1.7 & 2.7 & 2.7 & 0.3 & 11.8 & $4.37 \times 10^{2}$ & 7.0 & $\mathcal{D}_{\text {MBW }} B_{\operatorname{deg} 4}$ & 0.14 \\
\hline $39-61$ & $0.9<|\eta|<1.9$ & 7.2 & 3.3 & 6.1 & 5.2 & 1.3 & 29.2 & $9.70 \times 10^{2}$ & 4.0 & $\mathcal{D}_{\mathrm{MBW}} B_{\operatorname{deg} 4}$ & 0.31 \\
\hline $39-61$ & $|\eta|<0.9$ & 3.6 & 1.1 & 2.6 & 2.2 & 0.9 & 14.5 & $4.81 \times 10^{2}$ & 2.8 & $\mathcal{D}_{\mathrm{MBW}}$ & 0.26 \\
\hline $61-76$ & $1.9<|\eta|<2.4$ & 1.2 & 1.5 & 1.8 & 1.7 & 0.2 & 5.2 & $1.48 \times 10^{2}$ & 7.6 & $\mathcal{D}_{\mathrm{MBW}} B_{\operatorname{deg} 4}$ & 0.11 \\
\hline $61-76$ & $0.9<|\eta|<1.9$ & 4.8 & 3.6 & 4.5 & 4.4 & 0.7 & 20.3 & $5.12 \times 10^{2}$ & 4.2 & $\mathcal{D}_{\mathrm{MBW}} B_{\operatorname{deg} 4}$ & 0.29 \\
\hline $61-76$ & $|\eta|<0.9$ & 3.2 & 1.6 & 2.3 & 2.1 & 0.6 & 13.1 & $3.22 \times 10^{2}$ & 3.0 & $\mathcal{D}_{\mathrm{MBW}}$ & 0.28 \\
\hline $76-91$ & $1.9<|\eta|<2.4$ & 1.2 & 3.1 & 2.2 & 2.1 & 0.2 & 5.8 & $1.04 \times 10^{2}$ & 7.1 & $\mathcal{D}_{\mathrm{MBW}} B_{\operatorname{deg} 4}$ & 0.14 \\
\hline $76-91$ & $0.9<|\eta|<1.9$ & 4.4 & 8.7 & 6.2 & 6.0 & 1.1 & 20.3 & $3.60 \times 10^{2}$ & 4.2 & $\mathcal{D}_{\mathrm{MBW}} B_{\operatorname{deg} 4}$ & 0.35 \\
\hline $76-91$ & $|\eta|<0.9$ & 3.1 & 4.0 & 3.8 & 3.6 & 0.9 & 13.7 & $2.36 \times 10^{2}$ & 3.2 & $\mathcal{D}_{\mathrm{MBW}}$ & 0.34 \\
\hline $91-95$ & $|\eta|<2.4$ & 1.7 & 6.4 & 2.5 & 2.6 & 0.5 & 8.6 & 96.0 & 4.0 & $\mathcal{D}_{\mathrm{MBW}}$ & 0.28 \\
\hline $95-100$ & $|\eta|<2.4$ & 2.0 & 19 & 1.5 & 1.4 & 0.7 & 13.7 & 83.4 & 4.1 & $\mathcal{D}_{\text {MBW }}$ & 0.48 \\
\hline Total & $|\eta|<2.4$ & 59 & 61 & 51 & 52 & 49 & 253 & $1.30 \times 10^{4}$ & 3.9 & & \\
\hline
\end{tabular}


simulated dimuon invariant mass distribution for each production process in each category for $m_{H}=120,125$, and $130 \mathrm{GeV}$. The fit parameters are interpolated for masses within that range. The invariant mass distribution of the background primarily follows the smoothly falling spectrum of the high-mass DY background. Secondary contributions come from the single and pair production of top quarks. In each category, the background distribution is modeled by fitting the data with a single analytic function, chosen from a set of alternative options. These include a sum of exponential functions, Bernstein polynomials $\left(B_{\operatorname{deg} n}\right)$, and a modified version of the Breit-Wigner $\mathrm{Z}$ boson line shape $\mathcal{D}_{\mathrm{MBW}}$ derived and validated by fitting FEWZ predictions of the DY invariant mass distribution at next-to-NLO $[44,45]$ :

$$
\mathcal{D}_{\mathrm{MBW}}(x)=\frac{\mathrm{e}^{a_{2} x+a_{3} x^{2}}}{\left(x-m_{\mathrm{Z}}\right)^{a_{1}}+\left(\frac{\Gamma_{\mathrm{Z}}}{2}\right)^{a_{1}}},
$$

where $m_{\mathrm{Z}}$ and $\Gamma_{\mathrm{Z}}$ are the mass and the width, respectively, of the $\mathrm{Z}$ boson fixed to known values [46]. In addition, FEWZ spectra templates multiplied by polynomial functions are considered, as well as a modified Breit-Wigner distribution multiplied by a Bernstein polynomial of up to degree $4\left(B_{\operatorname{deg}} 4\right)$. The chosen function maximizes the expected sensitivity while introducing only a negligible bias in the measured signal yield, which is determined as follows. In each category, background-only fits to the data are performed with every function. From each of these fits, thousands of pseudodata sets are generated, taking into account the uncertainties in the fit parameters and their correlations, and simulated signal events are added according to their expected SM yields. Each of the background functions is then used to fit the pseudodata sets generated from every other function, with the total signal yield floating freely in the fit. The bias is estimated as the median excess or deficit in the measured signal yield relative to the SM expectation. Accepted functions in each category have a maximum possible bias of less than $20 \%$ of the statistical uncertainties for $m_{H}=120,125$, and $130 \mathrm{GeV}$. Including these deviations as spurious signals leads to an overall uncertainty in the calculated limit of less than $1 \%$, which is neglected. Correlation between bias terms is also found to be negligible.

The systematic uncertainties considered in the analysis account for possible mismodeling in the signal shape or rate. The shape of the reconstructed Higgs boson invariant mass is affected by the muon momentum scale and resolution. Uncertainties in the calibration of these values are propagated to the shape of the invariant mass distribution of the Higgs boson, assuming a Gaussian prior, yielding variations of up to $0.05 \%$ in the position of the peak and up to $10 \%$ in its width. Jet energy uncertainties in scale and resolution affect the analysis through migrations between categories. The largest variation of this kind amounts to $6 \%$ of the relative yield. Uncertainty in the simulation of additional pileup events is modeled by varying the total inelastic cross section $[47,48]$ by $\pm 5 \%$, which translates to $\approx 1 \%$ variations in the yields. The systematic uncertainty in the $b$ tagging or light-quark and gluon jet mistagging efficiencies results in event migration across categories of $\approx 1 \%$. Lepton efficiency mismodeling is accounted for with trigger and isolated muon identification uncertainties $(\approx 2 \%)$. The factorization and renormalization scales used in the MC simulations are varied up and down separately by a factor of 2 , translating to changes of up to $6 \%$ in the signal acceptance per category. The parton distribution functions used in the signal MC simulations are varied using the NNPDF3.0 replicas, which yield differences of $\approx 2 \%$. In the comparison of measured signal yields with expectation, additional uncertainties in the calculated signal cross sections are considered. They are due to the choice of factorization and renormalization scale $(3.9,0.4,3.8,1.9$, and $\lesssim 10 \%$, for $g g H, \mathrm{VBF}, Z H, W H$, and $t \bar{t} H$, respectively) and parton distribution functions $(3.2,2.1,1.6,1.9$, and $3.7 \%)$, as well as the $1.7 \%$ uncertainty in the $H \rightarrow \mu^{+} \mu^{-}$branching fraction [15]. Finally, a $2.5 \%$ uncertainty is associated with the integrated luminosity measurement [49].

A maximum likelihood signal-plus-background fit to the dimuon invariant mass spectrum is performed across all categories to measure the signal strength modifier $\mu$, defined as $(\sigma \mathcal{B})_{\mathrm{obs}} /(\sigma \mathcal{B})_{\mathrm{SM}}$ where $\sigma$ indicates the Higgs boson production cross section. The best fit signal strength for a Higgs boson mass hypothesis of $125.09 \mathrm{GeV}$ $\left(\hat{\mu}_{125}\right)$ and $68 \%$ C.L. interval is extracted with a profile likelihood ratio, according to the procedure described in Ref. [50], yielding $\hat{\mu}_{125}=0.7 \pm 1.0$ (stat $)_{-0.1}^{+0.2}$ (syst) for $m_{H}=125.09 \mathrm{GeV}$ [51]. Figure 2 shows the background component and the signal-plus-background fits to the data in all categories combined, weighted by the expected ratio of signal to signal plus background in each category. The 95\% C.L. upper limit on the signal strength modifier computed with the asymptotic $\mathrm{CL}_{\mathrm{s}}$ method [52-54] and the compatibility of the dimuon yield with the backgroundonly hypothesis for the 2016 data set $(13 \mathrm{TeV})$ are also derived. The observed (expected for $\mu=0$ ) upper limit at 95\% C.L. for $m_{H}=125.09 \mathrm{GeV}$ is 3.0 (2.5), with an observed (expected for $\mu=1$ ) significance of the incompatibility with the background-only hypothesis of $0.6(0.9)$ standard deviation (s.d.).

The 95\% C.L. upper limit on the signal strength as a function of $m_{H}$ in the region around the Higgs boson mass for a combination of data recorded at center-of-mass energies of 7, 8, and $13 \mathrm{TeV}$ is shown in Fig. 3 and yields an observed (expected for $\mu=0$ ) limit on the production rate of 2.9 (2.2) times the SM value at $m_{H}=125.09 \mathrm{GeV}$. The observed limit generally agrees well with the expected limit curve for $\mu=1$ that is also shown and corresponds to an upper limit on the $H \rightarrow \mu^{+} \mu^{-}$branching fraction of 


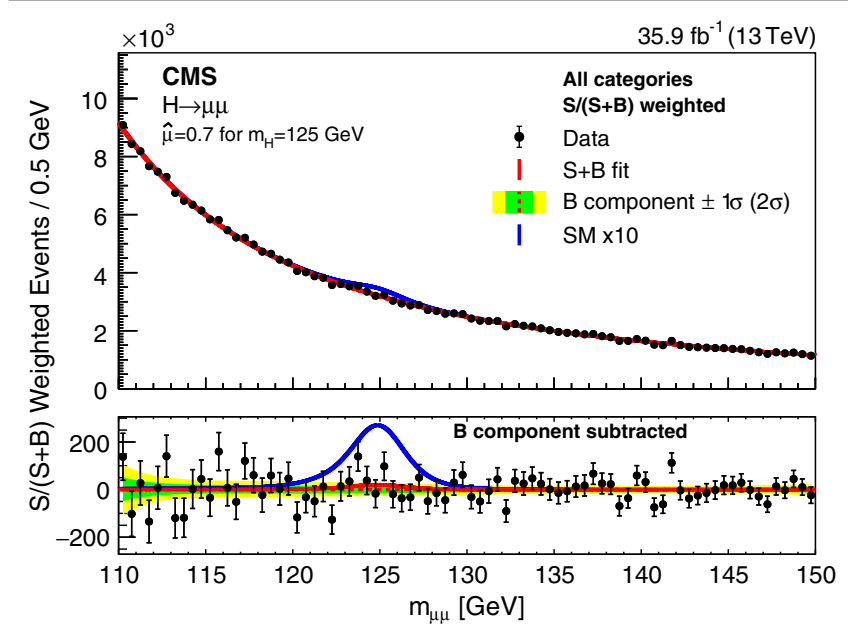

FIG. 2. Data and weighted sum of signal-plus-background fits to each category. Events are weighted according to the expected ratio of signal to signal plus background in the category to which they belong. The solid blue line represents the expectation from the SM Higgs boson multiplied by a factor of 10 . The lower panel shows the difference between the data and the background component of the fit.

$6.4 \times 10^{-4}$, assuming the SM production cross sections. The best fit signal strength for $m_{H}=125.09 \mathrm{GeV}$ is $\hat{\mu}_{125}^{\text {comb }}=1.0 \pm 1.0(\text { stat })_{-0.1}^{+0.1}$ (syst), and the observed combined significance is 0.9 s.d. The expected value for $\mu=1.0$ is $\hat{\mu}_{125}^{\mathrm{comb}}=1.0_{-1.0}^{+1.1}$, and the combined expected significance is 1.0 s.d. Theoretical uncertainties are considered correlated across the data sets, while the main experimental uncertainties are considered uncorrelated.

In summary, we present a search for the Higgs boson decaying to two muons using data recorded by the CMS experiment at the LHC in 2016 at a center-of-mass energy

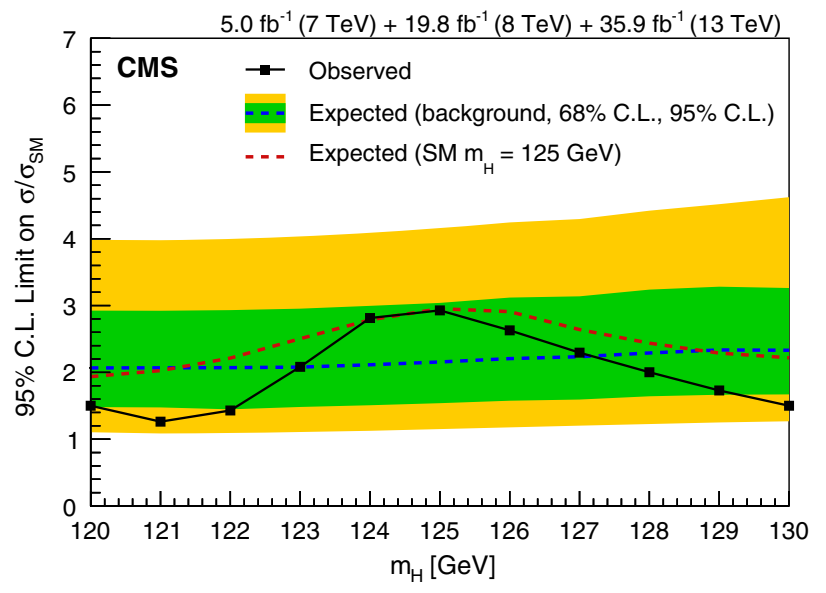

FIG. 3. The 95\% C.L. upper limit on the signal strength modifier $\mu$ in the region around the Higgs boson mass for the combination of the 7,8 , and $13 \mathrm{TeV}$ data sets together with the expected limit obtained in the background-only hypothesis (dashed black line) and in the signal-plus-background hypothesis (dashed red line) for the SM Higgs boson with $m_{H}=125 \mathrm{GeV}$. of $13 \mathrm{TeV}$, corresponding to an integrated luminosity of $35.9 \mathrm{fb}^{-1}$. No significant evidence for this decay is observed. Limits are set on the cross section times the branching fraction of the Higgs boson decaying to two muons. The combination with data recorded at center-ofmass energies of 7 and $8 \mathrm{TeV}$ yields a 95\% confidence level observed upper limit of 2.9 times the standard model value for $m_{H}=125.09 \mathrm{GeV}$. The corresponding expected upper limit in the absence of a SM decay in this channel is 2.2 , which is the most sensitive to date. This corresponds to an observed (standard model expected) significance of the Higgs boson decaying into two muons of 0.9 (1.0) standard deviation and an observed signal strength of $1.0 \pm 1.0$ (stat) \pm 0.1 (syst). Assuming standard model production cross sections for the Higgs boson, the observed limit corresponds to an upper limit of $6.4 \times 10^{-4}$ on the Higgs boson branching fraction to two muons.

We congratulate our colleagues in the CERN accelerator departments for the excellent performance of the LHC and thank the technical and administrative staffs at CERN and at other CMS institutes for their contributions to the success of the CMS effort. In addition, we gratefully acknowledge the computing centers and personnel of the Worldwide LHC Computing Grid for delivering so effectively the computing infrastructure essential to our analyses. Finally, we acknowledge the enduring support for the construction and operation of the LHC and the CMS detector provided by the following funding agencies: BMWFW and FWF (Austria); FNRS and FWO (Belgium); CNPq, CAPES, FAPERJ, and FAPESP (Brazil); MES (Bulgaria); CERN; CAS, MoST, and NSFC (China); COLCIENCIAS (Colombia); MSES and CSF (Croatia); RPF (Cyprus); SENESCYT (Ecuador); MoER, ERC IUT, and ERDF (Estonia); Academy of Finland, MEC, and HIP (Finland); CEA and CNRS/IN2P3 (France); BMBF, DFG, and HGF (Germany); GSRT (Greece); NKFIA (Hungary); DAE and DST (India); IPM (Iran); SFI (Ireland); INFN (Italy); MSIP and NRF (Republic of Korea); LAS (Lithuania); MOE and UM (Malaysia); BUAP, CINVESTAV, CONACYT, LNS, SEP, and UASLP-FAI (Mexico); MBIE (New Zealand); PAEC (Pakistan); MSHE and NSC (Poland); FCT (Portugal); JINR (Dubna); MON, RosAtom, RAS, and RFBR (Russia); MESTD (Serbia); SEIDI, CPAN, PCTI, and FEDER (Spain); Swiss Funding Agencies (Switzerland); MST (Taipei); ThEPCenter, IPST, STAR, and NSTDA (Thailand); TUBITAK and TAEK (Turkey); NASU and SFFR (Ukraine); STFC (United Kingdom); and DOE and NSF (USA).

[1] F. Englert and R. Brout, Broken Symmetry and the Mass of Gauge Vector Mesons, Phys. Rev. Lett. 13, 321 (1964).

[2] P. W. Higgs, Broken symmetries, massless particles and gauge fields, Phys. Lett. 12, 132 (1964). 
[3] P. W. Higgs, Broken Symmetries and the Masses of Gauge Bosons, Phys. Rev. Lett. 13, 508 (1964).

[4] P. W. Higgs, Spontaneous Symmetry Breakdown without Massless Bosons, Phys. Rev. 145, 1156 (1966).

[5] ATLAS Collaboration, Observation of a new particle in the search for the standard model Higgs boson with the ATLAS detector at the LHC, Phys. Lett. B 716, 1 (2012).

[6] CMS Collaboration, Observation of a new boson at a mass of $125 \mathrm{GeV}$ with the CMS experiment at the LHC, Phys. Lett. B 716, 30 (2012).

[7] CMS Collaboration, Observation of a new boson with mass near $125 \mathrm{GeV}$ in pp collisions at $\sqrt{s}=7$ and $8 \mathrm{TeV}$, J. High Energy Phys. 06 (2013) 081.

[8] CMS Collaboration, Evidence for the Higgs boson decay to a bottom quark-antiquark pair, Phys. Lett. B 780, 501 (2018).

[9] ATLAS Collaboration, Evidence for the $h \rightarrow b \bar{b}$ decay with the ATLAS detector, J. High Energy Phys. 12 (2017) 024.

[10] CMS Collaboration, Observation of the Higgs boson decay to a pair of $\tau$ leptons with the CMS detector, Phys. Lett. B 779, 283 (2018).

[11] ATLAS Collaboration, Evidence for the Higgs-boson Yukawa coupling to tau leptons with the ATLAS detector, J. High Energy Phys. 04 (2015) 117.

[12] CMS Collaboration, Observation of $t \bar{t} \mathrm{H}$ Production, Phys. Rev. Lett. 120, 231801 (2018).

[13] ATLAS Collaboration, Observation of Higgs boson production in association with a top quark pair at the LHC with the ATLAS detector, Phys. Lett. B 784, 173 (2018).

[14] ATLAS and CMS Collaborations, Combined Measurement of the Higgs Boson Mass in pp Collisions at $\sqrt{s}=7$ and $8 \mathrm{TeV}$ with the ATLAS and CMS Experiments, Phys. Rev. Lett. 114, 191803 (2015).

[15] LHC Higgs Cross Section Working Group, Handbook of LHC Higgs Cross Sections: 4. Deciphering the Nature of the Higgs Sector (CERN, Geneva, Switzerland, 2016).

[16] S. Dittmaier et al. (LHC Higgs Cross Section Working Group), Handbook of LHC Higgs Cross Sections: 1. Inclusive Observables, CERN Yellow Report: Monographs (CERN, Geneva, Switzerland, 2011).

[17] CMS Collaboration, Constraints on the Higgs boson width from off-shell production and decay to Z-boson pairs, Phys. Lett. B 736, 64 (2014).

[18] ATLAS Collaboration, Search for the standard model Higgs boson decay to $\mu^{+} \mu^{-}$with the ATLAS detector, Phys. Lett. B 738, 68 (2014).

[19] CMS Collaboration, Search for a standard model-like Higgs boson in the $\mu^{+} \mu^{-}$and $e^{+} e^{-}$decay channels at the LHC, Phys. Lett. B 744, 184 (2015).

[20] ATLAS Collaboration, Search for the Dimuon Decay of the Higgs Boson in $p p$ Collisions at $\sqrt{s}=13 \mathrm{TeV}$ with the ATLAS Detector, Phys. Rev. Lett. 119, 051802 (2017).

[21] CMS Collaboration, The CMS experiment at the CERN LHC, J. Instrum. 3, S08004 (2008).

[22] P. Nason, A new method for combining NLO QCD with shower Monte Carlo algorithms, J. High Energy Phys. 11 (2004) 040.

[23] R. D. Ball et al. (NNPDF Collaboration), Parton distributions for the LHC run II, J. High Energy Phys. 04 (2015) 040.

[24] J. Alwall, R. Frederix, S. Frixione, V. Hirschi, F. Maltoni, O. Mattelaer, H.-S. Shao, T. Stelzer, P. Torrielli, and M. Zaro,
The automated computation of tree-level and next-toleading order differential cross sections, and their matching to parton shower simulations, J. High Energy Phys. 07 (2014) 079.

[25] P. Artoisenet, R. Frederix, O. Mattelaer, and R. Rietkerk, Automatic spin-entangled decays of heavy resonances in Monte Carlo simulations, J. High Energy Phys. 03 (2013) 015.

[26] T. Sjöstrand, S. Mrenna, and P.Z. Skands, A brief introduction to PYTHIA 8.1, Comput. Phys. Commun. 178, 852 (2008).

[27] CMS Collaboration, Event generator tunes obtained from underlying event and multiparton scattering measurements, Eur. Phys. J. C 76, 155 (2016).

[28] S. Agostinelli et al. (GEANT4 Collaboration), GEANT4A simulation toolkit, Nucl. Instrum. Methods Phys. Res., Sect. A 506, 250 (2003).

[29] R. Gavin, Y. Li, F. Petriello, and S. Quackenbush, FEWZ 2.0: A code for hadronic $\mathrm{Z}$ production at next-to-next-toleading order, Comput. Phys. Commun. 182, 2388 (2011).

[30] M. Czakon and A. Mitov, Top++: A program for the calculation of the top-pair cross-section at hadron colliders, Comput. Phys. Commun. 185, 2930 (2014).

[31] M. Aliev, H. Lacker, U. Langenfeld, S. Moch, P. Uwer, and M. Wiedermann, HATHOR: Hadronic top and heavy quarks cross section calculator, Comput. Phys. Commun. 182, 1034 (2011).

[32] P. Kant, O. M. Kind, T. Kintscher, T. Lohse, T. Martini, S. Mölbitz, P. Rieck, and P. Uwer, HatHor for single top-quark production: Updated predictions and uncertainty estimates for single top-quark production in hadronic collisions, Comput. Phys. Commun. 191, 74 (2015).

[33] J. M. Campbell, R. K. Ellis, and C. Williams, Vector boson pair production at the LHC, J. High Energy Phys. 07 (2011) 018.

[34] CMS Collaboration, Particle-flow reconstruction and global event description with the CMS detector, J. Instrum. 12, P10003 (2017).

[35] CMS Collaboration, Performance of electron reconstruction and selection with the CMS detector in proton-proton collisions at $\sqrt{s}=8 \mathrm{TeV}$, J. Instrum. 10, P06005 (2015).

[36] CMS Collaboration, Performance of the CMS muon detector and reconstruction with pp collisions at $\sqrt{s}=13 \mathrm{TeV}$, J. Instrum. 13, P06015 (2018).

[37] M. Cacciari, G. P. Salam, and G. Soyez, The anti- $k_{T}$ jet clustering algorithm, J. High Energy Phys. 04 (2008) 063.

[38] M. Cacciari, G. P. Salam, and G. Soyez, FastJet user manual, Eur. Phys. J. C 72, 1896 (2012).

[39] CMS Collaboration, Jet algorithms performance in $13 \mathrm{TeV}$ data, CMS Physics Analysis Summary, CERN Report No. CMS-PAS-JME-16-003, 2017, https://cds.cern.ch/ record/2256875.

[40] CMS Collaboration, Identification of heavy-flavour jets with the CMS detector in pp collisions at $13 \mathrm{TeV}$, J. Instrum. 13, P05011 (2018).

[41] CMS Collaboration, The CMS trigger system, J. Instrum. 12, P01020 (2017).

[42] M. Cacciari and G. P. Salam, Pileup subtraction using jet areas, Phys. Lett. B 659, 119 (2008). 
[43] H. Voss, A. Höcker, J. Stelzer, and F. Tegenfeldt, TMVA, the toolkit for multivariate data analysis with ROOT, Proc. Sci., ACAT2007 (2007) 040.

[44] D. Bourilkov, Photon-induced background for dilepton searches and measurements in pp collisions at $13 \mathrm{TeV}$, arXiv:1606.00523.

[45] D. Bourilkov, Exploring the LHC landscape with dileptons, arXiv:1609.08994.

[46] C. Patrignani et al. (Particle Data Group), Review of particle physics, Chin. Phys. C 40, 100001 (2016).

[47] ATLAS Collaboration, Measurement of the Inelastic ProtonProton Cross Section at $\sqrt{s}=13 \mathrm{TeV}$ with the ATLAS Detector at the LHC, Phys. Rev. Lett. 117, 182002 (2016).

[48] CMS Collaboration, Measurement of the inelastic protonproton cross section at $\sqrt{s}=13 \mathrm{TeV}$, J. High Energy Phys. 07 (2018) 161.

[49] CMS Collaboration, CMS luminosity measurements for the 2016 data taking period, CMS Physics Analysis Summary,
CERN Report No. CMS-PAS-LUM-17-001, 2017, http:// cds.cern.ch/record/2257069.

[50] CMS Collaboration, Precise determination of the mass of the Higgs boson and tests of compatibility of its couplings with the standard model predictions using proton collisions at 7 and 8 TeV, Eur. Phys. J. C 75, 212 (2015).

[51] ATLAS and CMS Collaborations, Procedure for the LHC Higgs boson search combination in summer 2011, CERN, Technical Report Nos. CMS-NOTE-2011-005, ATL-PHYSPUB-2011-11, 2011, https://cds.cern.ch/record/1379837.

[52] T. Junk, Confidence level computation for combining searches with small statistics, Nucl. Instrum. Methods Phys. Res., Sect. A 434, 435 (1999).

[53] A. L. Read, Presentation of search results: The $\mathrm{CL}_{\mathrm{s}}$ technique, J. Phys. G 28, 2693 (2002).

[54] G. Cowan, K. Cranmer, E. Gross, and O. Vitells, Asymptotic formulae for likelihood-based tests of new physics, Eur. Phys. J. C 71, 1554 (2011); Erratum, Eur. Phys. J. C 73, 2501 (2013).

A. M. Sirunyan, ${ }^{1}$ A. Tumasyan, ${ }^{1}$ W. Adam, ${ }^{2}$ F. Ambrogi, ${ }^{2}$ E. Asilar, ${ }^{2}$ T. Bergauer, ${ }^{2}$ J. Brandstetter, ${ }^{2}$ E. Brondolin, ${ }^{2}$ M. Dragicevic, ${ }^{2}$ J. Erö, ${ }^{2}$ A. Escalante Del Valle, ${ }^{2}$ M. Flechl, ${ }^{2}$ R. Frühwirth, ${ }^{2, b}$ V. M. Ghete, ${ }^{2}$ J. Hrubec, ${ }^{2}$ M. Jeitler, ${ }^{2, b}$ N. Krammer, ${ }^{2}$ I. Krätschmer, ${ }^{2}$ D. Liko, ${ }^{2}$ T. Madlener, ${ }^{2}$ I. Mikulec, ${ }^{2}$ N. Rad, ${ }^{2}$ H. Rohringer, ${ }^{2}$ J. Schieck, ${ }^{2, b}$ R. Schöfbeck, ${ }^{2}$ M. Spanring, ${ }^{2}$ D. Spitzbart, ${ }^{2}$ A. Taurok, ${ }^{2}$ W. Waltenberger, ${ }^{2}$ J. Wittmann, ${ }^{2}$ C.-E. Wulz ${ }^{2, b}$ M. Zarucki, ${ }^{2}$ V. Chekhovsky, ${ }^{3}$ V. Mossolov, ${ }^{3}$ J. Suarez Gonzalez, ${ }^{3}$ E. A. De Wolf, ${ }^{4}$ D. Di Croce, ${ }^{4}$ X. Janssen, ${ }^{4}$ J. Lauwers, ${ }^{4}$ M. Pieters, ${ }^{4}$ M. Van De Klundert, ${ }^{4}$ H. Van Haevermaet, ${ }^{4}$ P. Van Mechelen, ${ }^{4}$ N. Van Remortel, ${ }^{4}$ S. Abu Zeid, ${ }^{5}$ F. Blekman, ${ }^{5}$ J. D'Hondt, ${ }^{5}$ I. De Bruyn, ${ }^{5}$ J. De Clercq, ${ }^{5}$ K. Deroover, ${ }^{5}$ G. Flouris, ${ }^{5}$ D. Lontkovskyi, ${ }^{5}$ S. Lowette, ${ }^{5}$ I. Marchesini, ${ }^{5}$ S. Moortgat, ${ }^{5}$ L. Moreels, ${ }^{5}$ Q. Python,${ }^{5}$ K. Skovpen, ${ }^{5}$ S. Tavernier, ${ }^{5}$ W. Van Doninck, ${ }^{5}$ P. Van Mulders,${ }^{5}$ I. Van Parijs, ${ }^{5}$ D. Beghin, ${ }^{6}$ B. Bilin, ${ }^{6}$ H. Brun, ${ }_{6}^{6}$ B. Clerbaux, ${ }^{6}$ G. De Lentdecker, ${ }^{6}$ H. Delannoy, ${ }^{6}$ B. Dorney, ${ }_{6}^{6}$ G. Fasanella, ${ }^{6}$ L. Favart, ${ }^{6}$ R. Goldouzian, ${ }^{6}$ A. Grebenyuk, ${ }^{6}$ A. K. Kalsi, ${ }^{6}$ T. Lenzi, ${ }^{6}$ J. Luetic, ${ }^{6}$ E. Starling, ${ }^{6}$ C. Vander Velde, ${ }^{6}$ P. Vanlaer, ${ }^{6}$ D. Vannerom, ${ }^{6}$ T. Cornelis, ${ }^{7}$ D. Dobur, ${ }^{7}$ A. Fagot, ${ }^{7}$ M. Gul, ${ }^{7}$ I. Khvastunov, ${ }^{7, c}$ D. Poyraz, ${ }^{7}$ C. Roskas, ${ }^{7}$ D. Trocino, ${ }^{7}$ M. Tytgat, ${ }^{7}$ W. Verbeke, ${ }^{7}$

B. Vermassen, ${ }^{7}$ M. Vit, ${ }^{7}$ N. Zaganidis ${ }^{7}$ H. Bakhshiansohi,${ }^{8}$ O. Bondu, ${ }^{8}$ S. Brochet, ${ }^{8}$ G. Bruno, ${ }^{8}$ C. Caputo, ${ }^{8}$ P. David, ${ }^{8}$ C. Delaere, ${ }^{8}$ M. Delcourt, ${ }^{8}$ B. Francois,${ }^{8}$ A. Giammanco, ${ }^{8}$ G. Krintiras, ${ }^{8}$ V. Lemaitre, ${ }^{8}$ A. Magitteri, ${ }^{8}$ A. Mertens, ${ }^{8}$ M. Musich, ${ }^{8}$ K. Piotrzkowski, ${ }^{8}$ A. Saggio, ${ }^{8}$ M. Vidal Marono, ${ }^{8}$ S. Wertz, ${ }^{8}$ J. Zobec, ${ }^{8}$ F. L. Alves, ${ }^{9}$ G. A. Alves, ${ }^{9}$ L. Brito, ${ }^{9}$ G. Correia Silva, ${ }^{9}$ C. Hensel, ${ }^{9}$ A. Moraes,${ }^{9}$ M. E. Pol, ${ }^{9}$ P. Rebello Teles, ${ }^{9}$ E. Belchior Batista Das Chagas, ${ }^{10}$ W. Carvalho, ${ }^{10}$ J. Chinellato, ${ }^{10, \mathrm{~d}}$ E. Coelho, ${ }^{10}$ E. M. Da Costa, ${ }^{10}$ G. G. Da Silveira, ${ }^{10, e}$ D. De Jesus Damiao, ${ }^{10}$ C. De Oliveira Martins, ${ }^{10}$ S. Fonseca De Souza, ${ }^{10}$ H. Malbouisson, ${ }^{10}$ D. Matos Figueiredo, ${ }^{10}$ M. Melo De Almeida, ${ }^{10}$ C. Mora Herrera, ${ }^{10}$ L. Mundim, ${ }^{10}$ H. Nogima, ${ }^{10}$ W. L. Prado Da Silva, ${ }^{10}$ L. J. Sanchez Rosas, ${ }^{10}$ A. Santoro, ${ }^{10}$ A. Sznajder,${ }^{10}$ M. Thiel, ${ }^{10}$ E. J. Tonelli Manganote, ${ }^{10, d}$ F. Torres Da Silva De Araujo, ${ }^{10}$ A. Vilela Pereira, ${ }^{10}$ S. Ahuja, ${ }^{11 a}$ C. A. Bernardes, ${ }^{11 a}$ L. Calligaris, ${ }^{11 \mathrm{a}}$ T. R. Fernandez Perez Tomei, ${ }^{11 \mathrm{a}}$ E. M. Gregores, ${ }^{11 \mathrm{a}, 11 \mathrm{~b}}$ P. G. Mercadante, ${ }^{11 \mathrm{a}, 11 \mathrm{~b}}$ S. F. Novaes, ${ }^{11 \mathrm{a}}$ Sandra S. Padula, ${ }^{11 \mathrm{a}}$ D. Romero Abad,${ }^{11 \mathrm{a}, 1 \mathrm{~b}}$ A. Aleksandrov, ${ }^{12}$ R. Hadjiiska, ${ }^{12}$ P. Iaydjiev, ${ }^{12}$ A. Marinov, ${ }^{12}$ M. Misheva, ${ }^{12}$ M. Rodozov, ${ }^{12}$ M. Shopova, ${ }^{12}$ G. Sultanov, ${ }^{12}$ A. Dimitrov, ${ }^{13}$ L. Litov, ${ }^{13}$ B. Pavlov, ${ }^{13}$ P. Petkov, ${ }^{13}$ W. Fang, ${ }^{14, \mathrm{f}}$ X. Gao,${ }^{14, \mathrm{f}}$ L. Yuan,${ }^{14}$ M. Ahmad,${ }^{15}$ J. G. Bian, ${ }^{15}$ G. M. Chen, ${ }^{15}$ H. S. Chen, ${ }^{15}$ M. Chen, ${ }^{15}$ Y. Chen, ${ }^{15}$ C. H. Jiang, ${ }^{15}$ D. Leggat, ${ }^{15}$ H. Liao, ${ }^{15}$ Z. Liu, ${ }^{15}$ F. Romeo, ${ }^{15}$ S. M. Shaheen, ${ }^{15}$ A. Spiezia, ${ }^{15}$ J. Tao, ${ }^{15}$ C. Wang, ${ }^{15}$ Z. Wang, ${ }^{15}$ E. Yazgan, ${ }^{15}$ H. Zhang,${ }^{15}$ J. Zhao, ${ }^{15}$ Y. Ban, ${ }^{16}$ G. Chen, ${ }^{16}$ J. Li, ${ }^{16}$ Q. Li, ${ }^{16}$ S. Liu, ${ }^{16}$ Y. Mao, ${ }^{16}$ S. J. Qian, ${ }^{16}$ D. Wang, ${ }^{16} \mathrm{Z}$. Xu, ${ }^{16}$ Y. Wang, ${ }^{17}$ C. Avila, ${ }^{18}$ A. Cabrera ${ }^{18}$ C. A. Carrillo Montoya,${ }^{18}$ L. F. Chaparro Sierra, ${ }^{18}$ C. Florez, ${ }^{18}$ C. F. González Hernández, ${ }^{18}$

M. A. Segura Delgado, ${ }^{18}$ B. Courbon, ${ }^{19}$ N. Godinovic, ${ }^{19}$ D. Lelas, ${ }^{19}$ I. Puljak, ${ }^{19}$ T. Sculac, ${ }^{19}$ Z. Antunovic,${ }^{20}$ M. Kovac, ${ }^{20}$ V. Brigljevic, ${ }^{21}$ D. Ferencek, ${ }^{21}$ K. Kadija, ${ }^{21}$ B. Mesic, ${ }^{21}$ A. Starodumov, ${ }^{21, g}$ T. Susa, ${ }^{21}$ M. W. Ather, ${ }^{22}$ A. Attikis, ${ }^{22}$ G. Mavromanolakis, ${ }^{22}$ J. Mousa, ${ }^{22}$ C. Nicolaou, ${ }^{22}$ F. Ptochos, ${ }^{22}$ P. A. Razis, ${ }^{22}$ H. Rykaczewski, ${ }^{22}$ M. Finger, ${ }^{23, h}$ M. Finger Jr., ${ }^{23, \mathrm{~h}}$ E. Ayala, ${ }^{24}$ E. Carrera Jarrin, ${ }^{25}$ H. Abdalla, ${ }^{26, \mathrm{i}}$ A. A. Abdelalim, ${ }^{26, j, \mathrm{k}}$ S. Khalil, ${ }^{26, \mathrm{k}}$ S. Bhowmik, ${ }^{27}$ A. Carvalho Antunes De Oliveira, ${ }^{27}$ R. K. Dewanjee, ${ }^{27}$ K. Ehataht, ${ }^{27}$ M. Kadastik,${ }^{27}$ L. Perrini, ${ }^{27}$ M. Raidal, ${ }^{27}$ C. Veelken, ${ }^{27}$ 
P. Eerola, ${ }^{28}$ H. Kirschenmann, ${ }^{28}$ J. Pekkanen, ${ }^{28}$ M. Voutilainen, ${ }^{28}$ J. Havukainen, ${ }^{29}$ J. K. Heikkilä, ${ }^{29}$ T. Järvinen, ${ }^{29}$ V. Karimäki, ${ }^{29}$ R. Kinnunen, ${ }^{29}$ T. Lampén, ${ }^{29}$ K. Lassila-Perini, ${ }^{29}$ S. Laurila, ${ }^{29}$ S. Lehti ${ }^{29}$ T. Lindén, ${ }^{29}$ P. Luukka, ${ }^{29}$ T. Mäenpää, ${ }^{29}$ H. Siikonen, ${ }^{29}$ E. Tuominen, ${ }^{29}$ J. Tuominiemi, ${ }^{29}$ T. Tuuva,${ }^{30}$ M. Besancon, ${ }^{31}$ F. Couderc,${ }^{31}$ M. Dejardin, ${ }^{31}$ D. Denegri, ${ }^{31}$ J. L. Faure, ${ }^{31}$ F. Ferri, ${ }^{31}$ S. Ganjour, ${ }^{31}$ A. Givernaud, ${ }^{31}$ P. Gras, ${ }^{31}$ G. Hamel de Monchenault, ${ }^{31}$ P. Jarry, ${ }^{31}$ C. Leloup, ${ }^{31}$ E. Locci, ${ }^{31}$ J. Malcles, ${ }^{31}$ G. Negro, ${ }^{31}$ J. Rander, ${ }^{31}$ A. Rosowsky, ${ }^{31}$ M. Ö. Sahin, ${ }^{31}$ M. Titov, ${ }^{31}$ A. Abdulsalam, ${ }^{32,1}$ C. Amendola, ${ }^{32}$ I. Antropov, ${ }^{32}$ F. Beaudette, ${ }^{32}$ P. Busson, ${ }^{32}$ C. Charlot,${ }^{32}$ R. Granier de Cassagnac, ${ }^{32}$ I. Kucher, ${ }^{32}$ S. Lisniak, ${ }^{32}$ A. Lobanov, ${ }^{32}$ J. Martin Blanco, ${ }^{32}$ M. Nguyen,${ }^{32}$ C. Ochando,${ }^{32}$ G. Ortona, ${ }^{32}$ P. Paganini, ${ }^{32}$ P. Pigard, ${ }^{32}$ R. Salerno, ${ }^{32}$ J. B. Sauvan, ${ }^{32}$ Y. Sirois, ${ }^{32}$ A. G. Stahl Leiton, ${ }^{32}$ Y. Yilmaz, ${ }^{32}$ A. Zabi, ${ }^{32}$ A. Zghiche, ${ }^{32}$ J.-L. Agram, ${ }^{33, m}$ J. Andrea, ${ }^{33}$ D. Bloch, ${ }^{33}$ J.-M. Brom, ${ }^{33}$ E. C. Chabert, ${ }^{33}$ V. Cherepanov, ${ }^{33}$ C. Collard, ${ }^{33}$ E. Conte, ${ }^{33, \mathrm{~m}}$ J.-C. Fontaine, ${ }^{33, \mathrm{~m}}$ D. Gelé, ${ }^{33}$ U. Goerlach, ${ }^{33}$ M. Jansová, ${ }^{33}$ A.-C. Le Bihan, ${ }^{33}$ N. Tonon, ${ }^{33}$ P. Van Hove, ${ }^{33}$ S. Gadrat,${ }^{34}$ S. Beauceron, ${ }^{35}$ C. Bernet, ${ }^{35}$ G. Boudoul,${ }^{35}$ N. Chanon, ${ }^{35}$ R. Chierici, ${ }^{35}$ D. Contardo, ${ }^{35}$ P. Depasse, ${ }^{35}$ H. El Mamouni, ${ }^{35}$ J. Fay, ${ }^{35}$ L. Finco,${ }^{35}$ S. Gascon, ${ }^{35}$ M. Gouzevitch, ${ }^{35}$ G. Grenier ${ }^{35}$ B. Ille, ${ }^{35}$ F. Lagarde, ${ }^{35}$ I. B. Laktineh ${ }^{35}$ H. Lattaud, ${ }^{35}$ M. Lethuillier, ${ }^{35}$ L. Mirabito, ${ }^{35}$ A. L. Pequegnot, ${ }^{35}$ S. Perries, ${ }^{35}$ A. Popov, ${ }^{35, n}$ V. Sordini, ${ }^{35}$ M. Vander Donckt, ${ }^{35}$ S. Viret, ${ }^{35}$ S. Zhang, ${ }^{35}$ A. Khvedelidze, ${ }^{36, h}$ Z. Tsamalaidze,${ }^{37, h}$ C. Autermann, ${ }^{38}$ L. Feld ${ }^{38}$ M. K. Kiesel,${ }^{38}$ K. Klein ${ }^{38}$ M. Lipinski, ${ }^{38}$ M. Preuten,${ }^{38}$ M. P. Rauch, ${ }^{38}$ C. Schomakers, ${ }^{38}$ J. Schulz, ${ }^{38}$ M. Teroerde, ${ }^{38}$ B. Wittmer, ${ }^{38}$ V. Zhukov, ${ }^{38, n}$ A. Albert, ${ }^{39}$ D. Duchardt, ${ }^{39}$ M. Endres, ${ }^{39}$ M. Erdmann, ${ }^{39}$ S. Erdweg, ${ }^{39}$ T. Esch, ${ }^{39}$ R. Fischer, ${ }^{39}$ S. Ghosh,${ }^{39}$ A. Güth, ${ }^{39}$ T. Hebbeker, ${ }^{39}$ C. Heidemann, ${ }^{39}$ K. Hoepfner, ${ }^{39}$ S. Knutzen, ${ }^{39}$ L. Mastrolorenzo, ${ }^{39}$ M. Merschmeyer, ${ }^{39}$ A. Meyer, ${ }^{39}$ P. Millet, ${ }^{39}$ S. Mukherjee, ${ }^{39}$ T. Pook, ${ }^{39}$ M. Radziej, ${ }^{39}$ H. Reithler, ${ }^{39}$ M. Rieger, ${ }^{39}$ F. Scheuch, ${ }^{39}$ A. Schmidt, ${ }^{39}$ D. Teyssier, ${ }^{39}$ S. Thüer, ${ }^{39}$ G. Flügge, ${ }^{40}$ O. Hlushchenko, ${ }^{40}$ B. Kargoll, ${ }^{40}$ T. Kress, ${ }^{40}$ A. Künsken, ${ }^{40}$ T. Müller ${ }^{40}$ A. Nehrkorn, ${ }^{40}$ A. Nowack, ${ }^{40}$ C. Pistone,${ }^{40}$ O. Pooth, ${ }^{40}$ H. Sert, ${ }^{40}$ A. Stahl, ${ }^{40,0}$ M. Aldaya Martin, ${ }^{41}$ T. Arndt,${ }^{41}$ C. Asawatangtrakuldee, ${ }^{41}$ I. Babounikau, ${ }^{41}$ K. Beernaert, ${ }^{41}$ O. Behnke, ${ }^{41}$ U. Behrens, ${ }^{41}$ A. Bermúdez Martínez, ${ }^{41}$ D. Bertsche, ${ }^{41}$ A. A. Bin Anuar, ${ }^{41}$ K. Borras, ${ }^{41, p}$ V. Botta ${ }^{41}$ A. Campbell, ${ }^{41}$ P. Connor, ${ }^{41}$ C. Contreras-Campana, ${ }^{41}$ F. Costanza, ${ }^{41}$ V. Danilov, ${ }^{41}$ A. De Wit, ${ }^{41}$ M. M. Defranchis, ${ }^{41}$ C. Diez Pardos, ${ }^{41}$ D. Domínguez Damiani, ${ }^{41}$ G. Eckerlin,${ }^{41}$ T. Eichhorn, ${ }^{41}$ A. Elwood, ${ }^{41}$ E. Eren, ${ }^{41}$ E. Gallo, ${ }^{41, q}$ A. Geiser, ${ }^{41}$ J. M. Grados Luyando, ${ }^{41}$ A. Grohsjean, ${ }^{41}$ P. Gunnellini, ${ }^{41}$ M. Guthoff,${ }^{41}$ A. Harb, ${ }^{41}$ J. Hauk, ${ }^{41}$ H. Jung, ${ }^{41}$ M. Kasemann, ${ }^{41}$ J. Keaveney, ${ }^{41}$ C. Kleinwort, ${ }^{41}$ J. Knolle, ${ }^{41}$ D. Krücker, ${ }^{41}$ W. Lange, ${ }^{41}$ A. Lelek, ${ }^{41}$ T. Lenz, ${ }^{41}$ K. Lipka, ${ }^{41}$ W. Lohmann, ${ }^{41, \mathrm{r}}$ R. Mankel, ${ }^{41}$ I.-A. Melzer-Pellmann, ${ }^{41}$ A. B. Meyer, ${ }^{41}$ M. Meyer,${ }^{41}$ M. Missiroli, ${ }^{41}$ G. Mittag, ${ }^{41}$ J. Mnich, ${ }^{41}$ V. Myronenko, ${ }^{41}$ S. K. Pflitsch, ${ }^{41}$ D. Pitzl, ${ }^{41}$ A. Raspereza, ${ }^{41}$ M. Savitskyi, ${ }^{41}$ P. Saxena, ${ }^{41}$ P. Schütze, ${ }^{41}$ C. Schwanenberger, ${ }^{41}$ R. Shevchenko, ${ }^{41}$ A. Singh,${ }^{41}$ N. Stefaniuk, ${ }^{41}$ H. Tholen, ${ }^{41}$ A. Vagnerini, ${ }^{41}$ G. P. Van Onsem, ${ }^{41}$ R. Walsh ${ }^{41}$ Y. Wen, ${ }^{41}$ K. Wichmann, ${ }^{41}$ C. Wissing,${ }^{41}$ O. Zenaiev, ${ }^{41}$ R. Aggleton, ${ }^{42}$ S. Bein, ${ }^{42}$ A. Benecke, ${ }^{42}$ V. Blobel, ${ }^{42}$ M. Centis Vignali, ${ }^{42}$ T. Dreyer, ${ }^{42}$ E. Garutti, ${ }^{42}$ D. Gonzalez, ${ }^{42}$ J. Haller, ${ }^{42}$ A. Hinzmann, ${ }^{42}$ M. Hoffmann, ${ }^{42}$ A. Karavdina, ${ }^{42}$ R. Klanner, ${ }^{42}$ R. Kogler, ${ }^{42}$ N. Kovalchuk, ${ }^{42}$ S. Kurz, ${ }^{42}$ V. Kutzner, ${ }^{42}$ J. Lange, ${ }^{42}$ D. Marconi, ${ }^{42}$ J. Multhaup, ${ }^{42}$ M. Niedziela, ${ }^{42}$ D. Nowatschin, ${ }^{42}$ A. Perieanu, ${ }^{42}$ A. Reimers, ${ }^{42}$ O. Rieger, ${ }^{42}$ C. Scharf, ${ }^{42}$ P. Schleper, ${ }^{42}$ S. Schumann, ${ }^{42}$ J. Schwandt, ${ }^{42}$ J. Sonneveld, ${ }^{42}$ H. Stadie, ${ }^{42}$ G. Steinbrück, ${ }^{42}$ F. M. Stober,${ }^{42}$ M. Stöver, ${ }^{42}$ D. Troendle, ${ }^{42}$ E. Usai, ${ }^{42}$ A. Vanhoefer, ${ }^{42}$ B. Vormwald, ${ }^{42}$ M. Akbiyik, ${ }^{43}$ C. Barth, ${ }^{43}$ M. Baselga, ${ }^{43}$ S. Baur, ${ }^{43}$ E. Butz, ${ }^{43}$ R. Caspart, ${ }^{43}$ T. Chwalek, ${ }^{43}$ F. Colombo, ${ }^{43}$ W. De Boer ${ }^{43}$ A. Dierlamm, ${ }^{43}$ N. Faltermann, ${ }^{43}$ B. Freund, ${ }^{43}$ M. Giffels, ${ }^{43}$ M. A. Harrendorf, ${ }^{43}$ F. Hartmann, ${ }^{43,0}$ S. M. Heindl, ${ }^{43}$ U. Husemann, ${ }^{43}$ F. Kassel, ${ }^{43,0}$ I. Katkov, ${ }^{43, n}$ S. Kudella, ${ }^{43}$ H. Mildner, ${ }^{43}$ M. U. Mozer, ${ }^{43}$ Th. Müller, ${ }^{43}$ M. Plagge, ${ }^{43}$ G. Quast, ${ }^{43}$ K. Rabbertz, ${ }^{43}$ M. Schröder, ${ }^{43}$ I. Shvetsov, ${ }^{43}$ G. Sieber, ${ }^{43}$ H. J. Simonis, ${ }^{43}$ R. Ulrich, ${ }^{43}$ S. Wayand, ${ }^{43}$ M. Weber, ${ }^{43}$ T. Weiler, ${ }^{43}$ S. Williamson, ${ }^{43}$ C. Wöhrmann, ${ }^{43}$ R. Wolf, ${ }^{43}$ G. Anagnostou, ${ }^{44}$ G. Daskalakis, ${ }^{44}$ T. Geralis, ${ }^{44}$ A. Kyriakis, ${ }^{44}$ D. Loukas, ${ }^{44}$ G. Paspalaki, ${ }^{44}$ I. Topsis-Giotis, ${ }^{44}$ G. Karathanasis, ${ }^{45}$ S. Kesisoglou, ${ }^{45}$ A. Panagiotou, ${ }^{45}$ N. Saoulidou, ${ }^{45}$ E. Tziaferi, ${ }^{45}$ K. Vellidis ${ }^{45}$ K. Kousouris ${ }^{46}$ I. Papakrivopoulos, ${ }^{46}$ Y. Tsipolitis, ${ }^{46}$ I. Evangelou, ${ }^{47}$ C. Foudas, ${ }^{47}$ P. Gianneios, ${ }^{47}$ P. Katsoulis, ${ }^{47}$ P. Kokkas, ${ }^{47}$ S. Mallios, ${ }^{47}$ N. Manthos, ${ }^{47}$ I. Papadopoulos,${ }^{47}$ E. Paradas,${ }^{47}$ J. Strologas, ${ }^{47}$ F. A. Triantis, ${ }^{47}$ D. Tsitsonis, ${ }^{47}$ M. Csanad, ${ }^{48}$ N. Filipovic, ${ }^{48}$ P. Major, ${ }^{48}$ M. I. Nagy, ${ }^{48}$ G. Pasztor, ${ }^{48}$ O. Surányi, ${ }^{48}$ G. I. Veres, ${ }^{48}$ G. Bencze,${ }^{49}$ C. Hajdu,${ }^{49}$ D. Horvath, ${ }^{49, s}$ Á. Hunyadi, ${ }^{49}$ F. Sikler, ${ }^{49}$ T. Á. Vámi, ${ }^{49}$ V. Veszpremi, ${ }^{49}$ G. Vesztergombi, ${ }^{49, a, t}$ N. Beni, ${ }^{50}$ S. Czellar, ${ }^{50}$ J. Karancsi ${ }^{50, \text { u }}$ A. Makovec,${ }^{50}$ J. Molnar, ${ }^{50}$ Z. Szillasi, ${ }^{50}$ M. Bartók, ${ }^{51, t}$ P. Raics ${ }^{51}$ Z. L. Trocsanyi,${ }^{51}$ B. Ujvari, ${ }^{51}$ S. Choudhury, ${ }^{52}$ J. R. Komaragiri, ${ }^{52}$ S. Bahinipati, ${ }^{53, v}$ P. Mal,${ }^{53}$ K. Mandal, ${ }^{53}$ A. Nayak,${ }^{53, w}$ D. K. Sahoo, ${ }^{53, v}$ S. K. Swain, ${ }^{53}$ S. Bansal, ${ }^{54}$ S. B. Beri, ${ }^{54}$ V. Bhatnagar, ${ }^{54}$ S. Chauhan,${ }^{54}$ R. Chawla, ${ }^{54}$ N. Dhingra, ${ }^{54}$ R. Gupta, ${ }^{54}$ A. Kaur, ${ }^{54}$ A. Kaur, ${ }^{54}$ M. Kaur, ${ }^{54}$ S. Kaur, ${ }^{54}$ R. Kumar, ${ }^{54}$ P. Kumari, ${ }^{54}$ M. Lohan, ${ }^{54}$ A. Mehta, ${ }^{54}$

S. Sharma, ${ }^{54}$ J. B. Singh, ${ }^{54}$ G. Walia, ${ }^{54}$ A. Bhardwaj, ${ }^{55}$ B. C. Choudhary, ${ }^{55}$ R. B. Garg, ${ }^{55}$ M. Gola, ${ }^{55}$ S. Keshri, ${ }^{55}$ Ashok Kumar, ${ }^{55}$ S. Malhotra, ${ }^{55}$ M. Naimuddin, ${ }^{55}$ P. Priyanka, ${ }^{55}$ K. Ranjan, ${ }^{55}$ Aashaq Shah, ${ }^{55}$ R. Sharma, ${ }^{55}$ R. Bhardwaj, ${ }^{56, x}$ 
M. Bharti, ${ }^{56}$ R. Bhattacharya, ${ }^{56}$ S. Bhattacharya, ${ }^{56}$ U. Bhawandeep, ${ }^{56, x}$ D. Bhowmik, ${ }^{56}$ S. Dey, ${ }^{56}$ S. Dutt, ${ }^{56, x}$ S. Dutta ${ }^{56}$ S. Ghosh, ${ }^{56}$ K. Mondal,${ }^{56}$ S. Nandan, ${ }^{56}$ A. Purohit,${ }^{56}$ P. K. Rout, ${ }^{56}$ A. Roy, ${ }^{56}$ S. Roy Chowdhury,${ }^{56}$ S. Sarkar,${ }^{56}$ M. Sharan,${ }^{56}$ B. Singh ${ }^{56}$ S. Thakur, ${ }^{56, x}$ P. K. Behera,${ }^{57}$ R. Chudasama, ${ }^{58}$ D. Dutta,${ }^{58}$ V. Jha,${ }^{58}$ V. Kumar, ${ }^{58}$ P. K. Netrakanti, ${ }^{58}$ L. M. Pant,${ }^{58}$ P. Shukla, ${ }^{58}$ T. Aziz, ${ }^{59}$ M. A. Bhat, ${ }^{59}$ S. Dugad, ${ }^{59}$ B. Mahakud, ${ }^{59}$ S. Mitra, ${ }^{59}$ G. B. Mohanty, ${ }^{59}$ N. Sur, ${ }^{59}$ B. Sutar, ${ }^{59}$ Ravindra Kumar Verma, ${ }^{59}$ S. Banerjee, ${ }^{60}$ S. Bhattacharya,${ }^{60}$ S. Chatterjee, ${ }^{60}$ P. Das, ${ }^{60}$ M. Guchait,${ }^{60}$ Sa. Jain, ${ }^{60}$ S. Kumar,${ }^{60}$ M. Maity, ${ }^{60, y}$ G. Majumder, ${ }^{60}$ K. Mazumdar,${ }^{60}$ N. Sahoo, ${ }^{60}$ T. Sarkar, ${ }^{60, y}$ S. Chauhan, ${ }^{61}$ S. Dube, ${ }^{61}$ V. Hegde, ${ }^{61}$ A. Kapoor, ${ }^{61}$

K. Kothekar, ${ }^{61}$ S. Pandey, ${ }^{61}$ A. Rane, ${ }^{61}$ S. Sharma ${ }^{61}$ S. Chenarani, ${ }^{62, z}$ E. Eskandari Tadavani, ${ }^{62}$ S. M. Etesami, ${ }^{62, z}$ M. Khakzad, ${ }^{62}$ M. Mohammadi Najafabadi, ${ }^{62}$ M. Naseri, ${ }^{62}$ F. Rezaei Hosseinabadi, ${ }^{62}$ B. Safarzadeh, ${ }^{62, a a}$ M. Zeinali, ${ }^{62}$ M. Felcini, ${ }^{63}$ M. Grunewald, ${ }^{63}$ M. Abbrescia, ${ }^{64 a, 64 b}$ C. Calabria, ${ }^{64 a, 64 b}$ A. Colaleo, ${ }^{64 a}$ D. Creanza, ${ }^{64 a, 64 c}$ L. Cristella, ${ }^{64 a, 64 b}$ N. De Filippis, ${ }^{64 a, 64 c}$ M. De Palma, ${ }^{64 a, 64 b}$ A. Di Florio, ${ }^{64 a, 64 b}$ F. Errico, ${ }^{64 a, 64 b}$ L. Fiore, ${ }^{64 a}$ A. Gelmi, ${ }^{64 a, 64 b}$ G. Iaselli, ${ }^{64 a, 64 c}$ S. Lezki, ${ }^{64,64 b}$ G. Maggi, ${ }^{64 a, 64 c}$ M. Maggi, ${ }^{64 a}$ G. Miniello, ${ }^{64 a, 64 b}$ S. My, ${ }^{64 a, 64 b}$ S. Nuzzo, ${ }^{64 a, 64 b}$ A. Pompili, ${ }^{64 a, 64 b}$ G. Pugliese ${ }^{64 a, 64 c}$ R. Radogna, ${ }^{64 a}$ A. Ranieri, ${ }^{64 a}$ G. Selvaggi, ${ }^{64 a, 64 b}$ A. Sharma, ${ }^{64 a}$ L. Silvestris,${ }^{64 a, 0}$ R. Venditti, ${ }^{64 a}$ P. Verwilligen, ${ }^{64 a}$ G. Zito, ${ }^{64 a}$ G. Abbiendi, ${ }^{65 a}$ C. Battilana ${ }^{65 a, 65 b}$ D. Bonacorsi, ${ }^{65 a, 65 b}$ L. Borgonovi, ${ }^{65 a, 65 b}$ S. Braibant-Giacomelli, ${ }^{65 a, 65 b}$ L. Brigliadori, ${ }^{65 a, 65 b}$ R. Campanini, ${ }^{65 a, 65 b}$ P. Capiluppi,${ }^{65 a, 65 b}$ A. Castro, ${ }^{65 a, 65 b}$ F. R. Cavallo, ${ }^{65 a}$ S. S. Chhibra, ${ }^{65,65 b}$ G. Codispoti, ${ }^{65 a, 65 b}$ M. Cuffiani, ${ }^{65 a, 65 b}$ G. M. Dallavalle, ${ }^{65 a}$ F. Fabbri, ${ }^{65 a}$ A. Fanfani, ${ }^{65 a, 65 b}$ P. Giacomelli, ${ }^{65 a}$ C. Grandi, ${ }^{65 a}$ L. Guiducci, ${ }^{65 a, 65 b}$ S. Marcellini, ${ }^{65 a}$ G. Masetti, ${ }^{65 a}$ A. Montanari, ${ }^{65 a}$ F. L. Navarria, ${ }^{65 a, 65 b}$ A. Perrotta, ${ }^{65 a}$ A. M. Rossi, ${ }^{65 a, 65 b}$ T. Rovelli, ${ }^{65 a, 65 b}$ G. P. Siroli, ${ }^{65,65 b}$ N. Tosi, ${ }^{65 a}$ S. Albergo, ${ }^{66 a, 66 b}$ A. Di Mattia, ${ }^{66 a}$ R. Potenza, ${ }^{66 a, 66 b}$ A. Tricomi, ${ }^{66 a, 66 b}$ C. Tuve, ${ }^{66 a, 66 b}$ G. Barbagli, ${ }^{67 a}$ K. Chatterjee, ${ }^{67 a, 67 b}$ V. Ciulli, ${ }^{67 a, 67 b}$ C. Civinini, ${ }^{67 a}$ R. D’Alessandro, ${ }^{67,67 b}$ E. Focardi, ${ }^{67 a, 67 b}$ G. Latino, ${ }^{67 a}$ P. Lenzi, ${ }^{67 a, 67 b}$ M. Meschini, ${ }^{67 a}$ S. Paoletti, ${ }^{67 a}$ L. Russo, ${ }^{67 a, b b}$ G. Sguazzoni, ${ }^{67 a}$ D. Strom, ${ }^{67 a}$ L. Viliani, ${ }^{67 a}$ L. Benussi, ${ }^{68}$ S. Bianco,${ }^{68}$ F. Fabbri, ${ }^{68}$ D. Piccolo, ${ }^{68}$ F. Primavera,${ }^{68,0}$ F. Ferro, ${ }^{69 a}$ F. Ravera, ${ }^{69 a, 69 b}$ E. Robutti, ${ }^{69 a}$ S. Tosi, ${ }^{69 a, 69 b}$ A. Benaglia, ${ }^{70 a}$ A. Beschi, ${ }^{70 a, 70 b}$ L. Brianza, ${ }^{70 a, 70 b}$ F. Brivio, ${ }^{70 a, 70 b}$ V. Ciriolo, ${ }^{70 a, 70 b, 0}$ M. E. Dinardo, ${ }^{70 a, 70 b}$ S. Fiorendi, ${ }^{70 a, 70 b}$ S. Gennai, ${ }^{70 a}$ A. Ghezzi, ${ }^{70 a, 70 b}$ P. Govoni, ${ }^{70 a, 70 b}$ M. Malberti ${ }^{70 a, 70 b}$ S. Malvezzi, ${ }^{70 a}$ R. A. Manzoni, ${ }^{70 a, 70 b}$ D. Menasce, ${ }^{70 a}$ L. Moroni,${ }^{70 a}$ M. Paganoni, ${ }^{70 a, 70 b}$ D. Pedrini, ${ }^{70 a}$ S. Pigazzini, ${ }^{70 a, 70 b}$ S. Ragazzi, ${ }^{70 a, 70 b}$ T. Tabarelli de Fatis, ${ }^{70 a, 70 b}$ S. Buontempo, ${ }^{71 \mathrm{a}}$ N. Cavallo, ${ }^{71 \mathrm{a}, 71 \mathrm{c}}$ A. Di Crescenzo, ${ }^{71 \mathrm{a}, 71 \mathrm{~b}}$ S. Di Guida, ${ }^{71 \mathrm{a}, 71 \mathrm{~d}, 0}$ F. Fabozzi, ${ }^{71 a, 71 c}$ F. Fienga, ${ }^{71 a, 71 b}$ G. Galati, ${ }^{71 a, 71 b}$ A. O. M. Iorio, ${ }^{71 a, 71 b}$ W. A. Khan, ${ }^{71 a}$ L. Lista, ${ }^{71 a}$ S. Meola, ${ }^{71 a, 71 d, o}$ P. Paolucci, ${ }^{71 a, 0}$ C. Sciacca, ${ }^{71 a, 71 b}$ E. Voevodina,${ }^{71 a, 71 b}$ P. Azzi, ${ }^{72 a}$ N. Bacchetta, ${ }^{72 a}$ L. Benato, ${ }^{72 a, 72 b}$ D. Bisello, ${ }^{72 a, 72 b}$ A. Boletti, ${ }^{72 a, 72 b}$ A. Bragagnolo, ${ }^{72 a}$ R. Carlin, ${ }^{72 a, 72 b}$ P. Checchia, ${ }^{72 a}$ M. Dall'Osso, ${ }^{72 a, 72 b}$ P. De Castro Manzano, ${ }^{72 a}$

T. Dorigo, ${ }^{72 a}$ U. Dosselli, ${ }^{72 a}$ F. Gasparini, ${ }^{72 a, 72 b}$ U. Gasparini, ${ }^{72 a, 72 b}$ A. Gozzelino, ${ }^{72 a}$ S. Lacaprara, ${ }^{72 a}$ P. Lujan, ${ }^{72 a}$ M. Margoni, ${ }^{72 a, 72 b}$ A. T. Meneguzzo ${ }^{72 a, 72 b}$ N. Pozzobon, ${ }^{72 a, 72 b}$ P. Ronchese, ${ }^{72 a, 72 b}$ R. Rossin, ${ }^{72 a, 72 b}$ F. Simonetto, ${ }^{72 a, 72 b}$ A. Tiko, ${ }^{72 a}$ E. Torassa, ${ }^{72 a}$ S. Ventura, ${ }^{72 a}$ M. Zanetti ${ }^{72 a, 72 b}$ A. Braghieri, ${ }^{73 a}$ A. Magnani, ${ }^{73 a}$ P. Montagna, ${ }^{73 a, 73 b}$ S. P. Ratti, ${ }^{73 a, 73 b}$ V. Re, ${ }^{73 a}$ M. Ressegotti, ${ }^{73 a, 73 b}$ C. Riccardi, ${ }^{73 a, 73 b}$ P. Salvini, ${ }^{73 a}$ I. Vai, ${ }^{73 a, 73 b}$ P. Vitulo, ${ }^{73 a, 73 b}$ L. Alunni Solestizi, ${ }^{74 a, 74 b}$ M. Biasini, ${ }^{74 a, 74 b}$ G. M. Bilei, ${ }^{74 a}$ C. Cecchi, ${ }^{74 a, 74 b}$ D. Ciangottini, ${ }^{74 a, 74 b}$ L. Fanò, ${ }^{74 a, 74 b}$ P. Lariccia, ${ }^{74 a, 74 b}$ E. Manoni, ${ }^{74 a}$ G. Mantovani, ${ }^{74 a, 74 b}$ V. Mariani, ${ }^{74 a, 74 b}$ M. Menichelli, ${ }^{74 a}$ A. Rossi, ${ }^{74 a, 74 b}$ A. Santocchia, ${ }^{74 a, 74 b}$ D. Spiga,${ }^{74 a}$ K. Androsov, ${ }^{75 a}$ P. Azzurri, ${ }^{75 a}$ G. Bagliesi, ${ }^{75 a}$ L. Bianchini, ${ }^{75 a}$ T. Boccali, ${ }^{75 a}$ L. Borrello, ${ }^{75 a}$ R. Castaldi, ${ }^{75 a}$ M. A. Ciocci, ${ }^{75 a, 75 b}$ R. Dell'Orso, ${ }^{75 a}$

G. Fedi, ${ }^{75 a}$ L. Giannini, ${ }^{75 a, 75 c}$ A. Giassi, ${ }^{75 a}$ M. T. Grippo, ${ }^{75 a}$ F. Ligabue, ${ }^{75 a, 75 c}$ E. Manca, ${ }^{75 a, 75 c}$ G. Mandorli, ${ }^{75 a, 75 c}$ A. Messineo, ${ }^{75 a, 75 b}$ F. Palla, ${ }^{75 a}$ A. Rizzi, ${ }^{75 a, 75 b}$ P. Spagnolo, ${ }^{75 a}$ R. Tenchini, ${ }^{75 a}$ G. Tonelli, ${ }^{75 a, 75 b}$ A. Venturi, ${ }^{75 a}$ P. G. Verdini, ${ }^{75 a}$ L. Barone,${ }^{76 a, 76 b}$ F. Cavallari, ${ }^{76 a}$ M. Cipriani, ${ }^{76 a, 76 b}$ N. Daci, ${ }^{76 a}$ D. Del Re $,{ }^{76 a}, 76 \mathrm{~b}$ E. Di Marco,${ }^{76 a, 76 b}$ M. Diemoz, ${ }^{76 a}$ S. Gelli, ${ }^{76 a, 76 b}$ E. Longo, ${ }^{76 a, 76 b}$ B. Marzocchi, ${ }^{76 a, 76 b}$ P. Meridiani, ${ }^{76 a}$ G. Organtini, ${ }^{76 a, 76 b}$ F. Pandolfi, ${ }^{76 a}$ R. Paramatti, ${ }^{76 a, 76 b}$ F. Preiato, ${ }^{76 a, 76 b}$ S. Rahatlou, ${ }^{76 a, 76 b}$ C. Rovelli, ${ }^{76 a}$ F. Santanastasio, ${ }^{76 a, 76 b}$ N. Amapane, ${ }^{77 a, 77 b}$ R. Arcidiacono, ${ }^{77 a, 77 c}$ S. Argiro, ${ }^{77 a, 77 b}$ M. Arneodo, ${ }^{77 a, 77 c}$ N. Bartosik,${ }^{77 a}$ R. Bellan, ${ }^{77 a, 77 b}$ C. Biino, ${ }^{77 a}$ N. Cartiglia,${ }^{77 a}$ F. Cenna,${ }^{77,77 b}$ M. Costa,${ }^{77,77 b}$ R. Covarelli, ${ }^{77,77 b}$ N. Demaria, ${ }^{77 a}$ B. Kiani, ${ }^{77,77 b}$ C. Mariotti, ${ }^{77 a}$ S. Maselli, ${ }^{77 a}$ E. Migliore,${ }^{77 a, 77 b}$ V. Monaco, ${ }^{77 a, 77 b}$ E. Monteil,${ }^{77,77 b}$ M. Monteno, ${ }^{77 a}$ M. M. Obertino, ${ }^{77 a, 77 b}$ L. Pacher, ${ }^{77 a, 77 b}$ N. Pastrone, ${ }^{77 a}$ M. Pelliccioni, ${ }^{77 a}$ G. L. Pinna Angioni, ${ }^{77 a, 77 b}$ A. Romero, ${ }^{77,77 b}$ M. Ruspa, ${ }^{77 a, 77 c}$ R. Sacchi,${ }^{77 a, 77 b}$ K. Shchelina, ${ }^{77 a, 77 b}$ V. Sola, ${ }^{77 a}$ A. Solano, ${ }^{77,77 b}$ A. Staiano, ${ }^{77 a}$ S. Belforte,${ }^{78 a}$ V. Candelise, ${ }^{78 a, 78 b}$ M. Casarsa, ${ }^{78 a}$ F. Cossutti, ${ }^{78 a}$ G. Della Ricca, ${ }^{78 a, 78 b}$ F. Vazzoler, ${ }^{78 a, 78 b}$ A. Zanetti, ${ }^{78 a}$ D. H. Kim, ${ }^{79}$ G. N. Kim, ${ }^{79}$ M. S. Kim,${ }^{79}$ J. Lee, ${ }^{79}$ S. Lee, ${ }^{79}$ S. W. Lee, ${ }^{79}$ C. S. Moon, ${ }^{79}$ Y. D. Oh ${ }^{79}$ S. Sekmen, ${ }^{79}$ D. C. Son,${ }^{79}$ Y. C. Yang,${ }^{79}$ H. Kim, ${ }^{80}$ D. H. Moon,${ }^{80}$ G. Oh, ${ }^{80}$ J. Goh, ${ }^{81}$ T. J. Kim, ${ }^{81}$ S. Cho, ${ }^{82}$ S. Choi ${ }^{82}$ Y. Go ${ }^{82}$ D. Gyun, ${ }^{82}$ S. Ha ${ }^{82}$ B. Hong ${ }^{82}$ Y. Jo, ${ }^{82}$ K. Lee, ${ }^{82}$ K. S. Lee, ${ }^{82}$ S. Lee, ${ }^{82}$ J. Lim, ${ }^{82}$ S. K. Park, ${ }^{82}$ Y. Roh, ${ }^{82}$ H. Kim, ${ }^{83}$ J. Almond ${ }^{84}$ J. Kim, ${ }^{84}$ J. S. Kim, ${ }^{84}$ H. Lee,${ }^{84}$ K. Lee, ${ }^{84}$ K. Nam, ${ }^{84}$ S. B. Oh ${ }^{84}$ B. C. Radburn-Smith,${ }^{84}$ S. h. Seo ${ }^{84}$ U. K. Yang, ${ }^{84}$ H. D. Yoo, ${ }^{84}$ G. B. Yu, ${ }^{84}$ H. Kim,${ }^{85}$ J. H. Kim, ${ }^{85}$ J. S. H. Lee, ${ }^{85}$ I. C. Park, ${ }^{85}$ Y. Choi, ${ }^{86}$ C. Hwang, ${ }^{86}$ J. Lee, ${ }^{86}$ 
I. Yu, ${ }^{86}$ V. Dudenas, ${ }^{87}$ A. Juodagalvis,${ }^{87}$ J. Vaitkus, ${ }^{87}$ I. Ahmed, ${ }^{88}$ Z. A. Ibrahim, ${ }^{88}$ M. A. B. Md Ali,,${ }^{88, c c}$ F. Mohamad Idris, ${ }^{88, d d}$ W. A. T. Wan Abdullah,${ }^{88}$ M. N. Yusli ${ }^{88}$ Z. Zolkapli, ${ }^{88}$ H. Castilla-Valdez ${ }^{89}$ E. De La Cruz-Burelo, ${ }^{89}$ M. C. Duran-Osuna, ${ }^{89}$ I. Heredia-De La Cruz, ${ }^{89, e e}$ R. Lopez-Fernandez, ${ }^{89}$ J. Mejia Guisao, ${ }^{89}$ R. I. Rabadan-Trejo, ${ }^{89}$ G. Ramirez-Sanchez, ${ }^{89}$ R Reyes-Almanza, ${ }^{89}$ A. Sanchez-Hernandez, ${ }^{89}$ S. Carrillo Moreno, ${ }^{90}$ C. Oropeza Barrera, ${ }^{90}$ F. Vazquez Valencia, ${ }^{90}$ J. Eysermans, ${ }^{91}$ I. Pedraza,${ }^{91}$ H. A. Salazar Ibarguen,${ }^{91}$ C. Uribe Estrada, ${ }^{91}$ A. Morelos Pineda, ${ }^{92}$ D. Krofcheck, ${ }^{93}$ S. Bheesette, ${ }^{94}$ P. H. Butler, ${ }^{94}$ A. Ahmad, ${ }^{95}$ M. Ahmad, ${ }^{95}$ M. I. Asghar, ${ }^{95}$ Q. Hassan, ${ }^{95}$ H. R. Hoorani, ${ }^{95}$ A. Saddique, ${ }^{95}$ M. A. Shah, ${ }^{95}$ M. Shoaib,${ }^{95}$ M. Waqas, ${ }^{95}$ H. Bialkowska, ${ }^{96}$ M. Bluj,${ }^{96}$ B. Boimska, ${ }^{96}$ T. Frueboes,${ }^{96}$ M. Górski ${ }^{96}$ M. Kazana ${ }^{96}$ K. Nawrocki,${ }^{96}$ M. Szleper, ${ }^{96}$ P. Traczyk, ${ }^{96}$ P. Zalewski, ${ }^{96}$ K. Bunkowski, ${ }^{97}$ A. Byszuk, ${ }^{97, f f}$ K. Doroba, ${ }^{97}$ A. Kalinowski, ${ }^{97}$ M. Konecki, ${ }^{97}$ J. Krolikowski, ${ }^{97}$ M. Misiura, ${ }^{97}$ M. Olszewski, ${ }^{97}$ A. Pyskir, ${ }^{97}$ M. Walczak, ${ }^{97}$ P. Bargassa, ${ }^{98}$ C. Beirão Da Cruz E Silva, ${ }^{98}$ A. Di Francesco,${ }^{98}$ P. Faccioli, ${ }^{98}$ B. Galinhas, ${ }^{98}$ M. Gallinaro, ${ }^{98}$ J. Hollar,${ }^{98}$ N. Leonardo, ${ }^{98}$ L. Lloret Iglesias, ${ }^{98}$ M. V. Nemallapudi, ${ }^{98}$ J. Seixas, ${ }^{98}$ G. Strong, ${ }^{98}$ O. Toldaiev, ${ }^{98}$ D. Vadruccio, ${ }^{98}$ J. Varela,${ }^{98}$ V. Alexakhin, ${ }^{99}$ A. Golunov, ${ }^{99}$ I. Golutvin, ${ }^{99}$ N. Gorbounov, ${ }^{99}$ I. Gorbunov, ${ }^{99}$ A. Kamenev ${ }^{99}$ V. Karjavin, ${ }^{99}$ A. Lanev, ${ }^{99}$ A. Malakhov, ${ }^{99}$ V. Matveev, ${ }^{99, g g, h h}$ P. Moisenz, ${ }^{99}$ V. Palichik, ${ }^{99}$ V. Perelygin,${ }^{99}$ M. Savina, ${ }^{99}$ S. Shmatov, ${ }^{99}$ S. Shulha, ${ }^{99}$ N. Skatchkov, ${ }^{99}$ V. Smirnov, ${ }^{99}$ A. Zarubin,,${ }^{99}$ V. Golovtsov, ${ }^{100}$ Y. Ivanov, ${ }^{100}$ V. Kim, ${ }^{100, i i}$ E. Kuznetsova, ${ }^{100, j j}$ P. Levchenko, ${ }^{100}$ V. Murzin, ${ }^{100}$ V. Oreshkin, ${ }^{100}$ I. Smirnov, ${ }^{100}$ D. Sosnov, ${ }^{100}$ V. Sulimov, ${ }^{100}$ L. Uvarov, ${ }^{100}$ S. Vavilov, ${ }^{100}$ A. Vorobyev,${ }^{100}$ Yu. Andreev, ${ }^{101}$ A. Dermenev, ${ }^{101}$ S. Gninenko, ${ }^{101}$ N. Golubev, ${ }^{101}$ A. Karneyeu, ${ }^{101}$ M. Kirsanov, ${ }^{101}$ N. Krasnikov, ${ }^{101}$

A. Pashenkov, ${ }^{101}$ D. Tlisov ${ }^{101}$ A. Toropin, ${ }^{101}$ V. Epshteyn, ${ }^{102}$ V. Gavrilov, ${ }^{102}$ N. Lychkovskaya,${ }^{102}$ V. Popov, ${ }^{102}$ I. Pozdnyakov, ${ }^{102}$ G. Safronov, ${ }^{102}$ A. Spiridonov, ${ }^{102}$ A. Stepennov, ${ }^{102}$ V. Stolin, ${ }^{102}$ M. Toms, ${ }^{102}$ E. Vlasov, ${ }^{102}$ A. Zhokin, ${ }^{102}$ T. Aushev, ${ }^{103}$ A. Bylinkin, ${ }^{103, \mathrm{hh}}$ R. Chistov, ${ }^{104, \mathrm{kk}}$ M. Danilov, ${ }^{104, \mathrm{kk}}$ P. Parygin, ${ }^{104}$ D. Philippov, ${ }^{104}$ S. Polikarpov, ${ }^{104}$ E. Tarkovskii, ${ }^{104}$ V. Andreev ${ }^{105}$ M. Azarkin, ${ }^{105, \text { hh }}$ I. Dremin, ${ }^{105, \text { hh }}$ M. Kirakosyan, ${ }^{105, \text { hh }}$ S. V. Rusakov, ${ }^{105}$ A. Terkulov, ${ }^{105}$ A. Baskakov, ${ }^{106}$ A. Belyaev, ${ }^{106}$ E. Boos, ${ }^{106}$ V. Bunichev, ${ }^{106}$ M. Dubinin, ${ }^{106,11}$ L. Dudko, ${ }^{106}$ A. Ershov,${ }^{106}$ A. Gribushin, ${ }^{106}$ V. Klyukhin, ${ }^{106}$ O. Kodolova, ${ }^{106}$ I. Lokhtin, ${ }^{106}$ I. Miagkov, ${ }^{106}$ S. Obraztsov, ${ }^{106}$ S. Petrushanko, ${ }^{106}$ V. Savrin, ${ }^{106}$ V. Blinov ${ }^{107, \mathrm{~mm}}$ T. Dimova, ${ }^{107, \mathrm{~mm}}$ L. Kardapoltsev, ${ }^{107, \mathrm{~mm}}$ D. Shtol, ${ }^{107, \mathrm{~mm}}$ Y. Skovpen, ${ }^{107, \mathrm{~mm}}$ I. Azhgirey,${ }^{108}$ I. Bayshev, ${ }^{108}$ S. Bitioukov, ${ }^{108}$ D. Elumakhov, ${ }^{108}$ A. Godizov, ${ }^{108}$ V. Kachanov, ${ }^{108}$ A. Kalinin, ${ }^{108}$ D. Konstantinov, ${ }^{108}$ P. Mandrik,${ }^{108}$ V. Petrov, ${ }^{108}$ R. Ryutin, ${ }^{108}$ S. Slabospitskii, ${ }^{108}$ A. Sobol, ${ }^{108}$ S. Troshin, ${ }^{108}$ N. Tyurin, ${ }^{108}$ A. Uzunian, ${ }^{108}$ A. Volkov, ${ }^{108}$ A. Babaev, ${ }^{109}$ P. Adzic, ${ }^{110, n n}$ P. Cirkovic, ${ }^{110}$ D. Devetak, ${ }^{110}$ M. Dordevic, ${ }^{110}$ J. Milosevic, ${ }^{110}$ J. Alcaraz Maestre, ${ }^{111}$ A. Álvarez Fernández, ${ }^{111}$ I. Bachiller, ${ }^{111}$ M. Barrio Luna, ${ }^{111}$ J. A. Brochero Cifuentes, ${ }^{111}$ M. Cerrada,${ }^{111}$ N. Colino, ${ }^{111}$ B. De La Cruz, ${ }^{111}$ A. Delgado Peris,${ }^{111}$ C. Fernandez Bedoya,${ }^{111}$ J. P. Fernández Ramos, ${ }^{111}$ J. Flix, ${ }^{111}$ M. C. Fouz, ${ }^{111}$

O. Gonzalez Lopez, ${ }^{111}$ S. Goy Lopez, ${ }^{111}$ J. M. Hernandez,${ }^{111}$ M. I. Josa, ${ }^{111}$ D. Moran, ${ }^{111}$ A. Pérez-Calero Yzquierdo, ${ }^{111}$ J. Puerta Pelayo, ${ }^{111}$ I. Redondo, ${ }^{111}$ L. Romero, ${ }^{111}$ M. S. Soares, ${ }^{111}$ A. Triossi, ${ }^{111}$ C. Albajar, ${ }^{112}$ J. F. de Trocóniz, ${ }^{112}$ J. Cuevas, ${ }^{113}$ C. Erice, ${ }^{113}$ J. Fernandez Menendez, ${ }^{113}$ S. Folgueras, ${ }^{113}$ I. Gonzalez Caballero, ${ }^{113}$ J. R. González Fernández, ${ }^{113}$ E. Palencia Cortezon, ${ }^{113}$ S. Sanchez Cruz, ${ }^{113}$ P. Vischia, ${ }^{113}$ J. M. Vizan Garcia, ${ }^{113}$ I. J. Cabrillo, ${ }^{114}$ A. Calderon, ${ }^{114}$

B. Chazin Quero, ${ }^{114}$ J. Duarte Campderros, ${ }^{114}$ M. Fernandez,${ }^{114}$ P. J. Fernández Manteca, ${ }^{114}$ A. García Alonso, ${ }^{114}$ J. Garcia-Ferrero, ${ }^{114}$ G. Gomez, ${ }^{114}$ A. Lopez Virto, ${ }^{114}$ J. Marco,${ }^{114}$ C. Martinez Rivero, ${ }^{114}$ P. Martinez Ruiz del Arbol, ${ }^{114}$ F. Matorras, ${ }^{114}$ J. Piedra Gomez,${ }^{114}$ C. Prieels, ${ }^{114}$ T. Rodrigo, ${ }^{114}$ A. Ruiz-Jimeno, ${ }^{114}$ L. Scodellaro, ${ }^{114}$ N. Trevisani, ${ }^{114}$ I. Vila, ${ }^{114}$ R. Vilar Cortabitarte, ${ }^{114}$ D. Abbaneo, ${ }^{115}$ B. Akgun, ${ }^{115}$ E. Auffray, ${ }^{115}$ P. Baillon, ${ }^{115}$ A. H. Ball, ${ }^{115}$ D. Barney, ${ }^{115}$ J. Bendavid, ${ }^{115}$ M. Bianco, ${ }^{115}$ A. Bocci, ${ }^{115}$ C. Botta, ${ }^{115}$ T. Camporesi, ${ }^{115}$ M. Cepeda, ${ }^{115}$ G. Cerminara, ${ }^{115}$ E. Chapon, ${ }^{115}$ Y. Chen, ${ }^{115}$ G. Cucciati, ${ }^{115}$ D. d'Enterria, ${ }^{115}$ A. Dabrowski, ${ }^{115}$ V. Daponte, ${ }^{115}$ A. David, ${ }^{115}$ A. De Roeck, ${ }^{115}$ N. Deelen, ${ }^{115}$ M. Dobson, ${ }^{115}$ T. du Pree, ${ }^{115}$ M. Dünser, ${ }^{115}$ N. Dupont, ${ }^{115}$ A. Elliott-Peisert, ${ }^{115}$ P. Everaerts, ${ }^{115}$ F. Fallavollita, ${ }^{115,00}$ G. Franzoni, ${ }^{115}$ J. Fulcher, ${ }^{115}$ W. Funk ${ }^{115}$ D. Gigi, ${ }^{115}$ A. Gilbert, ${ }^{115}$ K. Gill,,${ }^{115}$ F. Glege,${ }^{115}$ D. Gulhan,${ }^{115}$ J. Hegeman, ${ }^{115}$ V. Innocente, ${ }^{115}$ A. Jafari, ${ }^{115}$ P. Janot, ${ }^{115}$ O. Karacheban, ${ }^{115, \mathrm{r}}$ J. Kieseler, ${ }^{115}$ V. Knünz, ${ }^{115}$ A. Kornmayer, ${ }^{115}$ M. Krammer, ${ }^{15, b}$ C. Lange, ${ }^{115}$ P. Lecoq,${ }^{115}$ C. Lourenço, ${ }^{115}$ M. T. Lucchini, ${ }^{115}$ L. Malgeri, ${ }^{115}$ M. Mannelli, ${ }^{115}$ F. Meijers, ${ }^{115}$ J. A. Merlin, ${ }^{115}$ S. Mersi, ${ }^{115}$ E. Meschi, ${ }^{115}$ P. Milenovic, ${ }^{115, p p}$ F. Moortgat, ${ }^{115}$ M. Mulders, ${ }^{115}$ H. Neugebauer, ${ }^{115}$ J. Ngadiuba,${ }^{115}$

S. Orfanelli, ${ }^{115}$ L. Orsini, ${ }^{115}$ F. Pantaleo,${ }^{115,0}$ L. Pape,${ }^{115}$ E. Perez, ${ }^{115}$ M. Peruzzi,${ }^{115}$ A. Petrilli, ${ }^{115}$ G. Petrucciani, ${ }^{115}$ A. Pfeiffer, ${ }^{115}$ M. Pierini, ${ }^{115}$ F. M. Pitters, ${ }^{115}$ D. Rabady, ${ }^{115}$ A. Racz, ${ }^{115}$ T. Reis, ${ }^{115}$ G. Rolandi, ${ }^{115, q q}$ M. Rovere,${ }^{115}$ H. Sakulin, ${ }^{115}$ C. Schäfer, ${ }^{115}$ C. Schwick, ${ }^{115}$ M. Seidel,${ }^{115}$ M. Selvaggi, ${ }^{115}$ A. Sharma, ${ }^{115}$ P. Silva, ${ }^{115}$ P. Sphicas, ${ }^{115, \text { rr }}$ A. Stakia, ${ }^{115}$ J. Steggemann, ${ }^{115}$ M. Tosi, ${ }^{115}$ D. Treille, ${ }^{115}$ A. Tsirou, ${ }^{115}$ V. Veckalns,${ }^{115, s s}$ M. Verweij, ${ }^{115}$ W. D. Zeuner, ${ }^{115}$ W. Bertl, ${ }^{116, a}$ L. Caminada, ${ }^{16, t t}$ K. Deiters, ${ }^{116}$ W. Erdmann, ${ }^{116}$ R. Horisberger, ${ }^{116}$ Q. Ingram, ${ }^{116}$ H. C. Kaestli, ${ }^{116}$ D. Kotlinski, ${ }^{116}$ U. Langenegger, ${ }^{116}$ T. Rohe, ${ }^{116}$ S. A. Wiederkehr, ${ }^{116}$ M. Backhaus, ${ }^{117}$ L. Bäni, ${ }^{117}$ P. Berger,${ }^{117}$ B. Casal, ${ }^{117}$ 
N. Chernyavskaya, ${ }^{117}$ G. Dissertori, ${ }^{117}$ M. Dittmar, ${ }^{117}$ M. Donegà, ${ }^{117}$ C. Dorfer,${ }^{117}$ C. Grab, ${ }^{117}$ C. Heidegger, ${ }^{117}$ D. Hits, ${ }^{117}$ J. Hoss, ${ }^{117}$ T. Klijnsma, ${ }^{117}$ W. Lustermann, ${ }^{117}$ M. Marionneau, ${ }^{117}$ M. T. Meinhard,${ }^{117}$ D. Meister,${ }^{117}$ F. Micheli, ${ }^{117}$

P. Musella, ${ }^{117}$ F. Nessi-Tedaldi, ${ }^{117}$ J. Pata, ${ }^{117}$ F. Pauss, ${ }^{117}$ G. Perrin, ${ }^{117}$ L. Perrozzi, ${ }^{117}$ M. Quittnat, ${ }^{17}$ M. Reichmann,,${ }^{117}$ D. Ruini, ${ }^{117}$ D. A. Sanz Becerra, ${ }^{117}$ M. Schönenberger, ${ }^{117}$ L. Shchutska,${ }^{117}$ V. R. Tavolaro, ${ }^{117}$ K. Theofilatos,${ }^{117}$

M. L. Vesterbacka Olsson, ${ }^{117}$ R. Wallny, ${ }^{117}$ D. H. Zhu, ${ }^{117}$ T. K. Aarrestad, ${ }^{118}$ C. Amsler, ${ }^{118, \text { uu }}$ D. Brzhechko, ${ }^{118}$ M. F. Canelli, ${ }^{118}$ A. De Cosa, ${ }^{118}$ R. Del Burgo, ${ }^{118}$ S. Donato, ${ }^{118}$ C. Galloni, ${ }^{118}$ T. Hreus, ${ }^{118}$ B. Kilminster, ${ }^{118}$ I. Neutelings, ${ }^{118}$ D. Pinna, ${ }^{118}$ G. Rauco, ${ }^{118}$ P. Robmann, ${ }^{118}$ D. Salerno, ${ }^{118}$ K. Schweiger, ${ }^{118}$ C. Seitz, ${ }^{118}$ Y. Takahashi, ${ }^{118}$ A. Zucchetta, ${ }^{118}$ Y. H. Chang, ${ }^{119}$ K. y. Cheng, ${ }^{119}$ T. H. Doan, ${ }^{119}$ Sh. Jain, ${ }^{119}$ R. Khurana, ${ }^{119}$ C. M. Kuo, ${ }^{119}$ W. Lin, ${ }^{119}$ A. Pozdnyakov, ${ }^{119}$ S. S. Yu, ${ }^{119}$ P. Chang, ${ }^{120}$ Y. Chao, ${ }^{120}$ K. F. Chen, ${ }^{120}$ P. H. Chen, ${ }^{120}$ W.-S. Hou, ${ }^{120}$ Arun Kumar,${ }^{120}$ R.-S. Lu, ${ }^{120}$ E. Paganis, ${ }^{120}$ A. Psallidas, ${ }^{120}$ A. Steen, ${ }^{120}$ J. f. Tsai, ${ }^{120}$ B. Asavapibhop, ${ }^{121}$ N. Srimanobhas, ${ }^{121}$ N. Suwonjandee, ${ }^{121}$ A. Bat, ${ }^{122}$ F. Boran, ${ }^{122}$ S. Cerci, ${ }^{122, v v}$ S. Damarseckin, ${ }^{122}$ Z. S. Demiroglu, ${ }^{122}$ C. Dozen, ${ }^{122}$ I. Dumanoglu, ${ }^{122}$ S. Girgis, ${ }^{122}$ G. Gokbulut, ${ }^{122}$ Y. Guler, ${ }^{122}$ E. Gurpinar, ${ }^{122}$ I. Hos, ${ }^{122, \text { ww }}$ E. E. Kangal,${ }^{122, x x}$ O. Kara, ${ }^{122}$ A. Kayis Topaksu, ${ }^{122}$ U. Kiminsu, ${ }^{122}$ M. Oglakci, ${ }^{122}$ G. Onengut, ${ }^{122}$ K. Ozdemir, ${ }^{122, y y}$ S. Ozturk, ${ }^{122, z z}$ D. Sunar Cerci, ${ }^{122, v v}$ B. Tali, ${ }^{122, v v}$ U. G. Tok, ${ }^{122}$ S. Turkcapar, ${ }^{122}$ I. S. Zorbakir, ${ }^{122}$ C. Zorbilmez, ${ }^{122}$ B. Isildak, ${ }^{123, \text { aaa }}$ G. Karapinar, ${ }^{123, b b b}$ M. Yalvac, ${ }^{123}$ M. Zeyrek, ${ }^{123}$ I. O. Atakisi, ${ }^{124}$ E. Gülmez, ${ }^{124}$ M. Kaya, ${ }^{124, \mathrm{ccc}}$ O. Kaya, ${ }^{124, \text { ddd }}$ S. Ozkorucuklu, ${ }^{124, \text { eee }}$ S. Tekten, ${ }^{124}$ E. A. Yetkin, ${ }^{124, \text { fff }}$ M. N. Agaras, ${ }^{125}$ S. Atay, ${ }^{125}$ A. Cakir, ${ }^{125}$ K. Cankocak, ${ }^{125}$ Y. Komurcu, ${ }^{125}$ S. Sen, ${ }^{125, g g g}$ B. Grynyov, ${ }^{126}$ L. Levchuk, ${ }^{127}$ T. Alexander, ${ }^{128}$ F. Ball, ${ }^{128}$ L. Beck, ${ }^{128}$ J. J. Brooke, ${ }^{128}$ D. Burns, ${ }^{128}$ E. Clement, ${ }^{128}$ D. Cussans, ${ }^{128}$ O. Davignon, ${ }^{128}$ H. Flacher, ${ }^{128}$ J. Goldstein, ${ }^{128}$ G. P. Heath, ${ }^{128}$ H. F. Heath, ${ }^{128}$ L. Kreczko, ${ }^{128}$ D. M. Newbold, ${ }^{128, \text { thh }}$ S. Paramesvaran, ${ }^{128}$ B. Penning, ${ }^{128}$ T. Sakuma, ${ }^{128}$ D. Smith, ${ }^{128}$ V. J. Smith, ${ }^{128}$ J. Taylor, ${ }^{128}$ K. W. Bell, ${ }^{129}$ A. Belyaev, ${ }^{129, \text { iii }}$ C. Brew, ${ }^{129}$ R. M. Brown, ${ }^{129}$ D. Cieri, ${ }^{129}$ D. J. A. Cockerill, ${ }^{129}$ J. A. Coughlan, ${ }^{129}$ K. Harder, ${ }^{129}$ S. Harper, ${ }^{129}$ J. Linacre, ${ }^{129}$ E. Olaiya, ${ }^{129}$ D. Petyt, ${ }^{129}$ C. H. Shepherd-Themistocleous, ${ }^{129}$ A. Thea, ${ }^{129}$ I. R. Tomalin, ${ }^{129}$ T. Williams, ${ }^{129}$ W. J. Womersley, ${ }^{129}$ G. Auzinger, ${ }^{130}$ R. Bainbridge, ${ }^{130}$ P. Bloch, ${ }^{130}$ J. Borg, ${ }^{130}$ S. Breeze, ${ }^{130}$ O. Buchmuller, ${ }^{130}$ A. Bundock,${ }^{130}$ S. Casasso, ${ }^{130}$ D. Colling, ${ }^{130}$ L. Corpe,${ }^{130}$ P. Dauncey, ${ }^{130}$ G. Davies, ${ }^{130}$ M. Della Negra, ${ }^{130}$ R. Di Maria ${ }^{130}$ Y. Haddad,${ }^{130}$ G. Hall, ${ }^{130}$ G. Iles,${ }^{130}$ T. James, ${ }^{130}$ M. Komm, ${ }^{130}$ C. Laner, ${ }^{130}$ L. Lyons, ${ }^{130}$ A.-M. Magnan, ${ }^{130}$ S. Malik, ${ }^{130}$ A. Martelli, ${ }^{130}$ J. Nash, ${ }^{130, j j j}$ A. Nikitenko, ${ }^{130,8}$ V. Palladino, ${ }^{130}$ M. Pesaresi, ${ }^{130}$ A. Richards, ${ }^{130}$ A. Rose, ${ }^{130}$ E. Scott, ${ }^{130}$ C. Seez, ${ }^{130}$ A. Shtipliyski, ${ }^{130}$ G. Singh, ${ }^{130}$ M. Stoye, ${ }^{130}$ T. Strebler, ${ }^{130}$ S. Summers, ${ }^{130}$ A. Tapper, ${ }^{130}$ K. Uchida, ${ }^{130}$ T. Virdee, ${ }^{130,0}$ N. Wardle, ${ }^{130}$ D. Winterbottom, ${ }^{130}$ J. Wright, ${ }^{130}$ S. C. Zenz, ${ }^{130}$ J. E. Cole, ${ }^{131}$ P. R. Hobson, ${ }^{131}$ A. Khan, ${ }^{131}$ P. Kyberd,${ }^{131}$ C. K. Mackay, ${ }^{131}$ A. Morton, ${ }^{131}$ I. D. Reid, ${ }^{131}$ L. Teodorescu, ${ }^{131}$ S. Zahid, ${ }^{131}$ A. Borzou, ${ }^{132}$ K. Call, ${ }^{132}$ J. Dittmann, ${ }^{132}$ K. Hatakeyama ${ }^{132}$ H. Liu, ${ }^{132}$ C. Madrid, ${ }^{132}$ B. Mcmaster, ${ }^{132}$ N. Pastika, ${ }^{132}$ C. Smith, ${ }^{132}$ R. Bartek, ${ }^{133}$ A. Dominguez, ${ }^{133}$ A. Buccilli, ${ }^{134}$ S. I. Cooper, ${ }^{134}$ C. Henderson, ${ }^{134}$ P. Rumerio, ${ }^{134}$ C. West, ${ }^{134}$ D. Arcaro, ${ }^{135}$ T. Bose, ${ }^{135}$ D. Gastler, ${ }^{135}$ D. Rankin, ${ }^{135}$ C. Richardson, ${ }^{135}$ J. Rohlf, ${ }^{135}$ L. Sulak, ${ }^{135}$ D. Zou, ${ }^{135}$ G. Benelli, ${ }^{136}$ X. Coubez, ${ }^{136}$ D. Cutts, ${ }^{136}$ M. Hadley,${ }^{136}$ J. Hakala, ${ }^{136}$ U. Heintz, ${ }^{136}$ J. M. Hogan, ${ }^{136, k k k}$ K. H. M. Kwok, ${ }^{136}$ E. Laird, ${ }^{136}$ G. Landsberg, ${ }^{136}$ J. Lee, ${ }^{136}$ Z. Mao, ${ }^{136}$ M. Narain, ${ }^{136}$ J. Pazzini, ${ }^{136}$ S. Piperov, ${ }^{136}$ S. Sagir, ${ }^{136}$ R. Syarif, ${ }^{136}$ D. Yu, ${ }^{136}$ R. Band,${ }^{137}$ C. Brainerd, ${ }^{137}$ R. Breedon, ${ }^{137}$ D. Burns,${ }^{137}$ M. Calderon De La Barca Sanchez, ${ }^{137}$ M. Chertok, ${ }^{137}$ J. Conway ${ }^{137}$ R. Conway,${ }^{137}$ P. T. Cox, ${ }^{137}$ R. Erbacher, ${ }^{137}$ C. Flores, ${ }^{137}$ G. Funk, ${ }^{137}$ W. Ko, ${ }^{137}$ O. Kukral, ${ }^{137}$ R. Lander, ${ }^{137}$ C. Mclean, ${ }^{137}$ M. Mulhearn, ${ }^{137}$ D. Pellett,,${ }^{137}$ J. Pilot, ${ }^{137}$ S. Shalhout, ${ }^{137}$ M. Shi, ${ }^{137}$ D. Stolp, ${ }^{137}$ D. Taylor, ${ }^{137}$ K. Tos, ${ }^{137}$ M. Tripathi, ${ }^{137}$ Z. Wang, ${ }^{137}$ F. Zhang, ${ }^{137}$ M. Bachtis, ${ }^{138}$ C. Bravo, ${ }^{138}$ R. Cousins, ${ }^{138}$ A. Dasgupta, ${ }^{138}$ A. Florent ${ }^{138}$ J. Hauser, ${ }^{138}$ M. Ignatenko, ${ }^{138}$ N. Mccoll, ${ }^{138}$ S. Regnard, ${ }^{138}$ D. Saltzberg, ${ }^{138}$ C. Schnaible, ${ }^{138}$ V. Valuev, ${ }^{138}$ E. Bouvier, ${ }^{139}$ K. Burt, ${ }^{139}$ R. Clare, ${ }^{139}$ J. W. Gary, ${ }^{139}$ S. M. A. Ghiasi Shirazi, ${ }^{139}$ G. Hanson, ${ }^{139}$ G. Karapostoli, ${ }^{139}$ E. Kennedy, ${ }^{139}$ F. Lacroix, ${ }^{139}$ O. R. Long, ${ }^{139}$ M. Olmedo Negrete, ${ }^{139}$ M. I. Paneva, ${ }^{139}$ W. Si, ${ }^{139}$ L. Wang, ${ }^{139}$ H. Wei, ${ }^{139}$ S. Wimpenny, ${ }^{139}$ B. R. Yates, ${ }^{139}$ J. G. Branson, ${ }^{140}$ S. Cittolin, ${ }^{140}$ M. Derdzinski, ${ }^{140}$ R. Gerosa, ${ }^{140}$ D. Gilbert, ${ }^{140}$ B. Hashemi, ${ }^{140}$ A. Holzner, ${ }^{140}$ D. Klein, ${ }^{140}$ G. Kole, ${ }^{140}$ V. Krutelyov, ${ }^{140}$ J. Letts, ${ }^{140}$ M. Masciovecchio, ${ }^{140}$ D. Olivito, ${ }^{140}$ S. Padhi, ${ }^{140}$ M. Pieri, ${ }^{140}$ M. Sani, ${ }^{140}$ V. Sharma, ${ }^{140}$ S. Simon,,${ }^{140}$ M. Tadel, ${ }^{140}$ A. Vartak, ${ }^{140}$ S. Wasserbaech, ${ }^{140,111}$ J. Wood, ${ }^{140}$ F. Würthwein, ${ }^{140}$ A. Yagil,${ }^{140}$ G. Zevi Della Porta, ${ }^{140}$ N. Amin,${ }^{141}$ R. Bhandari, ${ }^{141}$ J. Bradmiller-Feld,${ }^{141}$

C. Campagnari, ${ }^{141}$ M. Citron, ${ }^{141}$ A. Dishaw, ${ }^{141}$ V. Dutta, ${ }^{141}$ M. Franco Sevilla, ${ }^{141}$ L. Gouskos, ${ }^{141}$ R. Heller, ${ }^{141}$ J. Incandela, ${ }^{141}$ A. Ovcharova, ${ }^{141}$ H. Qu, ${ }^{141}$ J. Richman, ${ }^{141}$ D. Stuart, ${ }^{141}$ I. Suarez, ${ }^{141}$ S. Wang, ${ }^{141}$ J. Yoo, ${ }^{141}$ D. Anderson, ${ }^{142}$ A. Bornheim, ${ }^{142}$ J. Bunn, ${ }^{142}$ J. M. Lawhorn, ${ }^{142}$ H. B. Newman, ${ }^{142}$ T. Q. Nguyen, ${ }^{142}$ M. Spiropulu, ${ }^{142}$ J. R. Vlimant, ${ }^{142}$ R. Wilkinson, ${ }^{142}$ S. Xie, ${ }^{142}$ Z. Zhang, ${ }^{142}$ R. Y. Zhu, ${ }^{142}$ M. B. Andrews, ${ }^{143}$ T. Ferguson, ${ }^{143}$ T. Mudholkar, ${ }^{143}$ M. Paulini, ${ }^{143}$ M. Sun, ${ }^{143}$ I. Vorobiev, ${ }^{143}$ M. Weinberg, ${ }^{143}$ J. P. Cumalat, ${ }^{144}$ W. T. Ford, ${ }^{144}$ F. Jensen, ${ }^{144}$ A. Johnson, ${ }^{144}$ M. Krohn, ${ }^{144}$ S. Leontsinis, ${ }^{144}$ E. MacDonald, ${ }^{144}$ T. Mulholland, ${ }^{144}$ K. Stenson, ${ }^{144}$ K. A. Ulmer, ${ }^{144}$ S. R. Wagner, ${ }^{144}$ J. Alexander, ${ }^{145}$ 
J. Chaves, ${ }^{145}$ Y. Cheng, ${ }^{145}$ J. Chu, ${ }^{145}$ A. Datta, ${ }^{145}$ K. Mcdermott, ${ }^{145}$ N. Mirman, ${ }^{145}$ J. R. Patterson, ${ }^{145}$ D. Quach, ${ }^{145}$ A. Rinkevicius, ${ }^{145}$ A. Ryd, ${ }^{145}$ L. Skinnari, ${ }^{145}$ L. Soffi, ${ }^{145}$ S. M. Tan ${ }^{145}$ Z. Tao ${ }^{145}$ J. Thom, ${ }^{145}$ J. Tucker, ${ }^{145}$ P. Wittich, ${ }^{145}$ M. Zientek, ${ }^{145}$ S. Abdullin, ${ }^{146}$ M. Albrow, ${ }^{146}$ M. Alyari, ${ }^{146}$ G. Apollinari, ${ }^{146}$ A. Apresyan, ${ }^{146}$ A. Apyan, ${ }^{146}$ S. Banerjee, ${ }^{146}$ L. A. T. Bauerdick, ${ }^{146}$ A. Beretvas, ${ }^{146}$ J. Berryhill, ${ }^{146}$ P. C. Bhat, ${ }^{146}$ G. Bolla ${ }^{146, a}$ K. Burkett, ${ }^{146}$ J. N. Butler, ${ }^{146}$ A. Canepa, ${ }^{146}$ G. B. Cerati, ${ }^{146}$ H. W. K. Cheung, ${ }^{146}$ F. Chlebana, ${ }^{146}$ M. Cremonesi, ${ }^{146}$ J. Duarte,${ }^{146}$ V. D. Elvira, ${ }^{146}$ J. Freeman, ${ }^{146}$ Z. Gecse, ${ }^{146}$ E. Gottschalk, ${ }^{146}$ L. Gray, ${ }^{146}$ D. Green, ${ }^{146}$ S. Grünendahl, ${ }^{146}$ O. Gutsche, ${ }^{146}$ J. Hanlon, ${ }^{146}$ R. M. Harris, ${ }^{146}$ S. Hasegawa, ${ }^{146}$ J. Hirschauer, ${ }^{146}$ Z. Hu, ${ }^{146}$ B. Jayatilaka, ${ }^{146}$ S. Jindariani, ${ }^{146}$ M. Johnson, ${ }^{146}$ U. Joshi, ${ }^{146}$ B. Klima, ${ }^{146}$ M. J. Kortelainen, ${ }^{146}$ B. Kreis, ${ }^{146}$ S. Lammel, ${ }^{146}$ D. Lincoln, ${ }^{146}$ R. Lipton, ${ }^{146}$ M. Liu, ${ }^{146}$ T. Liu, ${ }^{146}$ J. Lykken, ${ }^{146}$ K. Maeshima, ${ }^{146}$ N. Magini, ${ }^{146}$ J. M. Marraffino, ${ }^{146}$ D. Mason, ${ }^{146}$ P. McBride,${ }^{146}$ P. Merkel, ${ }^{146}$ S. Mrenna, ${ }^{146}$ S. Nahn, ${ }^{146}$ V. O’Dell, ${ }^{146}$ K. Pedro, ${ }^{146}$ C. Pena, ${ }^{146}$ O. Prokofyev, ${ }^{146}$ G. Rakness, ${ }^{146}$ L. Ristori, ${ }^{146}$ A. Savoy-Navarro, ${ }^{146, m m m}$ B. Schneider, ${ }^{146}$ E. Sexton-Kennedy, ${ }^{146}$ A. Soha, ${ }^{146}$ W. J. Spalding, ${ }^{146}$ L. Spiegel, ${ }^{146}$ S. Stoynev, ${ }^{146}$ J. Strait, ${ }^{146}$ N. Strobbe, ${ }^{146}$ L. Taylor, ${ }^{146}$ S. Tkaczyk,${ }^{146}$ N. V. Tran, ${ }^{146}$ L. Uplegger, ${ }^{146}$ E. W. Vaandering, ${ }^{146}$ C. Vernieri, ${ }^{146}$ M. Verzocchi, ${ }^{146}$ R. Vidal, ${ }^{146}$ M. Wang, ${ }^{146}$ H. A. Weber, ${ }^{146}$ A. Whitbeck, ${ }^{146}$ D. Acosta, ${ }^{147}$ P. Avery, ${ }^{147}$ P. Bortignon, ${ }^{147}$ D. Bourilkov, ${ }^{147}$ A. Brinkerhoff, ${ }^{147}$ L. Cadamuro, ${ }^{147}$ A. Carnes, ${ }^{147}$ M. Carver, ${ }^{147}$ D. Curry, ${ }^{147}$ R. D. Field, ${ }^{147}$ S. V. Gleyzer, ${ }^{147}$ B. M. Joshi, ${ }^{147}$ J. Konigsberg, ${ }^{147}$ A. Korytov, ${ }^{147}$ P. Ma,${ }^{147}$ K. Matchev,${ }^{147}$ H. Mei,${ }^{147}$ G. Mitselmakher, ${ }^{147}$ K. Shi,${ }^{147}$ D. Sperka, ${ }^{147}$ L. Thomas, ${ }^{147}$ J. Wang, ${ }^{147}$ S. Wang, ${ }^{147}$ Y. R. Joshi, ${ }^{148}$ S. Linn, ${ }^{148}$ A. Ackert, ${ }^{149}$ T. Adams, ${ }^{149}$ A. Askew, ${ }^{149}$ S. Hagopian, ${ }^{149}$ V. Hagopian, ${ }^{149}$ K. F. Johnson, ${ }^{149}$ T. Kolberg, ${ }^{149}$ G. Martinez, ${ }^{149}$ T. Perry, ${ }^{149}$ H. Prosper, ${ }^{149}$ A. Saha, ${ }^{149}$ A. Santra, ${ }^{149}$ V. Sharma, ${ }^{149}$ R. Yohay, ${ }^{149}$ M. M. Baarmand, ${ }^{150}$ V. Bhopatkar, ${ }^{150}$ S. Colafranceschi, ${ }^{150}$ M. Hohlmann, ${ }^{150}$ D. Noonan, ${ }^{150}$ T. Roy, ${ }^{150}$ F. Yumiceva, ${ }^{150}$ M. R. Adams, ${ }^{151}$ L. Apanasevich, ${ }^{151}$ D. Berry, ${ }^{151}$ R. R. Betts, ${ }^{151}$ R. Cavanaugh, ${ }^{151}$ X. Chen, ${ }^{151}$ S. Dittmer, ${ }^{151}$

O. Evdokimov, ${ }^{151}$ C. E. Gerber, ${ }^{151}$ D. A. Hangal, ${ }^{151}$ D. J. Hofman, ${ }^{151}$ K. Jung, ${ }^{151}$ J. Kamin,${ }^{151}$ C. Mills, ${ }^{151}$

I. D. Sandoval Gonzalez, ${ }^{151}$ M. B. Tonjes, ${ }^{151}$ N. Varelas, ${ }^{151}$ H. Wang, ${ }^{151}$ Z. Wu, ${ }^{151}$ J. Zhang, ${ }^{151}$ M. Alhusseini, ${ }^{152}$ B. Bilki, ${ }^{152, \text { nnn }}$ W. Clarida, ${ }^{152}$ K. Dilsiz, ${ }^{152,000}$ S. Durgut, ${ }^{152}$ R. P. Gandrajula, ${ }^{152}$ M. Haytmyradov, ${ }^{152}$ V. Khristenko, ${ }^{152}$ J.-P. Merlo, ${ }^{152}$ A. Mestvirishvili, ${ }^{152}$ A. Moeller, ${ }^{152}$ J. Nachtman, ${ }^{152}$ H. Ogul, ${ }^{152, p p p}$ Y. Onel, ${ }^{152}$ F. Ozok, ${ }^{152, q q q}$ A. Penzo, ${ }^{152}$ C. Snyder, ${ }^{152}$ E. Tiras, ${ }^{152}$ J. Wetzel,${ }^{152}$ B. Blumenfeld, ${ }^{153}$ A. Cocoros ${ }^{153}$ N. Eminizer, ${ }^{153}$ D. Fehling, ${ }^{153}$ L. Feng, ${ }^{153}$ A. V. Gritsan, ${ }^{153}$ W. T. Hung, ${ }^{153}$ P. Maksimovic,${ }^{153}$ J. Roskes, ${ }^{153}$ U. Sarica ${ }^{153}$ M. Swartz, ${ }^{153}$ M. Xiao, ${ }^{153}$ C. You, ${ }^{153}$ A. Al-bataineh, ${ }^{154}$ P. Baringer, ${ }^{154}$ A. Bean, ${ }^{154}$ S. Boren, ${ }^{154}$ J. Bowen, ${ }^{154}$ J. Castle, ${ }^{154}$ S. Khalil, ${ }^{154}$ A. Kropivnitskaya, ${ }^{154}$ D. Majumder, ${ }^{154}$ W. Mcbrayer, ${ }^{154}$ M. Murray, ${ }^{154}$ C. Rogan, ${ }^{154}$ S. Sanders, ${ }^{154}$ E. Schmitz, ${ }^{154}$ J. D. Tapia Takaki, ${ }^{154}$ Q. Wang, ${ }^{154}$ A. Ivanov, ${ }^{155}$ K. Kaadze ${ }^{155}$ Y. Maravin, ${ }^{155}$ A. Modak, ${ }^{155}$ A. Mohammadi, ${ }^{155}$ L. K. Saini, ${ }^{155}$ N. Skhirtladze, ${ }^{155}$ F. Rebassoo, ${ }^{156}$ D. Wright, ${ }^{156}$ A. Baden, ${ }^{157}$ O. Baron, ${ }^{157}$ A. Belloni, ${ }^{157}$ S. C. Eno, ${ }^{157}$ Y. Feng, ${ }^{157}$ C. Ferraioli, ${ }^{157}$ N. J. Hadley, ${ }^{157}$ S. Jabeen, ${ }^{157}$ G. Y. Jeng, ${ }^{157}$ R. G. Kellogg ${ }^{157}$ J. Kunkle, ${ }^{157}$ A. C. Mignerey, ${ }^{157}$ F. Ricci-Tam, ${ }^{157}$ Y. H. Shin, ${ }^{157}$ A. Skuja, ${ }^{157}$ S. C. Tonwar, ${ }^{157}$ K. Wong, ${ }^{157}$ D. Abercrombie, ${ }^{158}$ B. Allen, ${ }^{158}$ V. Azzolini, ${ }^{158}$ R. Barbieri, ${ }^{158}$ A. Baty, ${ }^{158}$ G. Bauer, ${ }^{158}$ R. Bi, ${ }^{158}$ S. Brandt, ${ }^{158}$ W. Busza, ${ }^{158}$ I. A. Cali, ${ }^{158}$ M. D'Alfonso, ${ }^{158}$ Z. Demiragli, ${ }^{158}$ G. Gomez Ceballos, ${ }^{158}$ M. Goncharov, ${ }^{158}$ P. Harris, ${ }^{158}$ D. Hsu, ${ }^{158}$ M. Hu, ${ }^{158}$ Y. Iiyama,${ }^{158}$ G. M. Innocenti, ${ }^{158}$ M. Klute,${ }^{158}$ D. Kovalskyi, ${ }^{158}$ Y.-J. Lee, ${ }^{158}$ A. Levin, ${ }^{158}$ P. D. Luckey, ${ }^{158}$ B. Maier, ${ }^{158}$ A. C. Marini, ${ }^{158}$ C. Mcginn, ${ }^{158}$ C. Mironov, ${ }^{158}$ S. Narayanan, ${ }^{158}$ X. Niu, ${ }^{158}$ C. Paus, ${ }^{158}$ C. Roland,${ }^{158}$ G. Roland, ${ }^{158}$ G. S. F. Stephans, ${ }^{158}$ K. Sumorok, ${ }^{158}$ K. Tatar, ${ }^{158}$ D. Velicanu, ${ }^{158}$ J. Wang, ${ }^{158}$ T. W. Wang, ${ }^{158}$ B. Wyslouch, ${ }^{158}$ S. Zhaozhong, ${ }^{158}$ A. C. Benvenuti, ${ }^{159}$ R. M. Chatterjee, ${ }^{159}$ A. Evans, ${ }^{159}$ P. Hansen, ${ }^{159}$ S. Kalafut, ${ }^{159}$ Y. Kubota, ${ }^{159}$ Z. Lesko, ${ }^{159}$ J. Mans, ${ }^{159}$ S. Nourbakhsh, ${ }^{159}$ N. Ruckstuhl, ${ }^{159}$ R. Rusack, ${ }^{159}$ J. Turkewitz, ${ }^{159}$ M. A. Wadud, ${ }^{159}$ J. G. Acosta, ${ }^{160}$ S. Oliveros, ${ }^{160}$ E. Avdeeva, ${ }^{161}$ K. Bloom, ${ }^{161}$ D. R. Claes, ${ }^{161}$ C. Fangmeier, ${ }^{161}$ F. Golf, ${ }^{161}$ R. Gonzalez Suarez, ${ }^{161}$ R. Kamalieddin, ${ }^{161}$ I. Kravchenko, ${ }^{161}$ J. Monroy, ${ }^{161}$ J. E. Siado, ${ }^{161}$ G. R. Snow, ${ }^{161}$ B. Stieger, ${ }^{161}$ A. Godshalk, ${ }^{162}$ C. Harrington, ${ }^{162}$ I. Iashvili, ${ }^{162}$ A. Kharchilava,${ }^{162}$ D. Nguyen, ${ }^{162}$ A. Parker, ${ }^{162}$ S. Rappoccio, ${ }^{162}$ B. Roozbahani, ${ }^{162}$ G. Alverson, ${ }^{163}$ E. Barberis, ${ }^{163}$ C. Freer, ${ }^{163}$ A. Hortiangtham, ${ }^{163}$ A. Massironi, ${ }^{163}$ D. M. Morse, ${ }^{163}$ T. Orimoto, ${ }^{163}$ R. Teixeira De Lima, ${ }^{163}$ T. Wamorkar, ${ }^{163}$ B. Wang, ${ }^{163}$ A. Wisecarver, ${ }^{163}$ D. Wood, ${ }^{163}$ S. Bhattacharya, ${ }^{164}$ O. Charaf,${ }^{164}$ K. A. Hahn, ${ }^{164}$ N. Mucia, ${ }^{164}$ N. Odell, ${ }^{164}$ M. H. Schmitt, ${ }^{164}$ K. Sung, ${ }^{164}$ M. Trovato, ${ }^{164}$ M. Velasco, ${ }^{164}$ R. Bucci, ${ }^{165}$ N. Dev, ${ }^{165}$ M. Hildreth,${ }^{165}$ K. Hurtado Anampa,${ }^{165}$ C. Jessop, ${ }^{165}$ D. J. Karmgard, ${ }^{165}$ N. Kellams, ${ }^{165}$ K. Lannon, ${ }^{165}$ W. Li, ${ }^{165}$ N. Loukas, ${ }^{165}$ N. Marinelli, ${ }^{165}$ F. Meng, ${ }^{165}$ C. Mueller, ${ }^{165}$ Y. Musienko, ${ }^{165, g g}$ M. Planer, ${ }^{165}$ A. Reinsvold, ${ }^{165}$ R. Ruchti, ${ }^{165}$ P. Siddireddy, ${ }^{165}$ G. Smith, ${ }^{165}$ S. Taroni, ${ }^{165}$ M. Wayne, ${ }^{165}$ A. Wightman, ${ }^{165}$ M. Wolf, ${ }^{165}$ A. Woodard, ${ }^{165}$ J. Alimena, ${ }^{166}$ L. Antonelli, ${ }^{166}$ B. Bylsma ${ }^{166}$ L. S. Durkin, ${ }^{166}$ S. Flowers, ${ }^{166}$ B. Francis, ${ }^{166}$ A. Hart, ${ }^{166}$ C. Hill, ${ }^{166}$ W. Ji, ${ }^{166}$ T. Y. Ling, ${ }^{166}$ W. Luo, ${ }^{166}$ B. L. Winer,${ }^{166}$ H. W. Wulsin, ${ }^{166}$ S. Cooperstein, ${ }^{167}$ P. Elmer, ${ }^{167}$ J. Hardenbrook, ${ }^{167}$ P. Hebda,${ }^{167}$ S. Higginbotham, ${ }^{167}$ A. Kalogeropoulos, ${ }^{167}$ D. Lange, ${ }^{167}$ J. Luo, ${ }^{167}$ D. Marlow, ${ }^{167}$ K. Mei, ${ }^{167}$ 
I. Ojalvo, ${ }^{167}$ J. Olsen, ${ }^{167}$ C. Palmer, ${ }^{167}$ P. Piroué ${ }^{167}$ J. Salfeld-Nebgen, ${ }^{167}$ D. Stickland, ${ }^{167}$ C. Tully, ${ }^{167}$ S. Malik, ${ }^{168}$ S. Norberg, ${ }^{168}$ A. Barker, ${ }^{169}$ V. E. Barnes, ${ }^{169}$ S. Das, ${ }^{169}$ L. Gutay, ${ }^{169}$ M. Jones,${ }^{169}$ A. W. Jung,${ }^{169}$ A. Khatiwada, ${ }^{169}$ D. H. Miller, ${ }^{169}$ N. Neumeister, ${ }^{169}$ C. C. Peng, ${ }^{169}$ H. Qiu, ${ }^{169}$ J. F. Schulte,${ }^{169}$ J. Sun, ${ }^{169}$ F. Wang, ${ }^{169}$ R. Xiao, ${ }^{169}$ W. Xie, ${ }^{169}$ T. Cheng, ${ }^{170}$ J. Dolen, ${ }^{170}$ N. Parashar,${ }^{170}$ Z. Chen, ${ }^{171}$ K. M. Ecklund, ${ }^{171}$ S. Freed, ${ }^{171}$ F. J. M. Geurts, ${ }^{171}$ M. Guilbaud,${ }^{171}$ M. Kilpatrick, ${ }^{171}$ W. Li, ${ }^{171}$ B. Michlin, ${ }^{171}$ B. P. Padley, ${ }^{171}$ J. Roberts, ${ }^{171}$ J. Rorie, ${ }^{171}$ W. Shi, ${ }^{171}$ Z. Tu, ${ }^{171}$ J. Zabel, ${ }^{171}$ A. Zhang, ${ }^{171}$ A. Bodek, ${ }^{172}$ P. de Barbaro, ${ }^{172}$ R. Demina, ${ }^{172}$ Y.t. Duh, ${ }^{172}$ J. L. Dulemba, ${ }^{172}$ C. Fallon, ${ }^{172}$ T. Ferbel, ${ }^{172}$ M. Galanti, ${ }^{172}$ A. Garcia-Bellido, ${ }^{172}$ J. Han,${ }^{172}$ O. Hindrichs, ${ }^{172}$ A. Khukhunaishvili,,${ }^{172}$ K. H. Lo, ${ }^{172}$ P. Tan, ${ }^{172}$ R. Taus, ${ }^{172}$ M. Verzetti, ${ }^{172}$ A. Agapitos, ${ }^{173}$ J. P. Chou, ${ }^{173}$ Y. Gershtein, ${ }^{173}$ T. A. Gómez Espinosa, ${ }^{173}$ E. Halkiadakis, ${ }^{173}$ M. Heindl, ${ }^{173}$ E. Hughes ${ }^{173}$ S. Kaplan, ${ }^{173}$ R. Kunnawalkam Elayavalli, ${ }^{173}$ S. Kyriacou, ${ }^{173}$ A. Lath, ${ }^{173}$ R. Montalvo, ${ }^{173}$ K. Nash, ${ }^{173}$ M. Osherson, ${ }^{173}$ H. Saka, ${ }^{173}$ S. Salur, ${ }^{173}$ S. Schnetzer, ${ }^{173}$ D. Sheffield, ${ }^{173}$ S. Somalwar, ${ }^{173}$ R. Stone, ${ }^{173}$ S. Thomas, ${ }^{173}$ P. Thomassen, ${ }^{173}$ M. Walker, ${ }^{173}$ A. G. Delannoy, ${ }^{174}$ J. Heideman, ${ }^{174}$ G. Riley, ${ }^{174}$ K. Rose, ${ }^{174}$ S. Spanier, ${ }^{174}$ K. Thapa, ${ }^{174}$ O. Bouhali, ${ }^{175, \text { rrr }}$ A. Castaneda Hernandez, ${ }^{175, \text { rrr }}$ A. Celik, ${ }^{175}$ M. Dalchenko, ${ }^{175}$ M. De Mattia, ${ }^{175}$ A. Delgado, ${ }^{175}$

S. Dildick, ${ }^{175}$ R. Eusebi, ${ }^{175}$ J. Gilmore, ${ }^{175}$ T. Huang, ${ }^{175}$ T. Kamon, ${ }^{175, \text { sss }}$ R. Mueller, ${ }^{175}$ Y. Pakhotin, ${ }^{175}$ R. Patel,${ }^{175}$ A. Perloff, ${ }^{175}$ L. Perniè, ${ }^{175}$ D. Rathjens, ${ }^{175}$ A. Safonov, ${ }^{175}$ A. Tatarinov, ${ }^{175}$ N. Akchurin, ${ }^{176}$ J. Damgov, ${ }^{176}$ F. De Guio, ${ }^{176}$ P. R. Dudero, ${ }^{176}$ J. Faulkner, ${ }^{176}$ S. Kunori,,${ }^{176}$ K. Lamichhane, ${ }^{176}$ S. W. Lee, ${ }^{176}$ T. Mengke, ${ }^{176}$ S. Muthumuni, ${ }^{176}$ T. Peltola, ${ }^{176}$ S. Undleeb, ${ }^{176}$ I. Volobouev, ${ }^{176}$ Z. Wang, ${ }^{176}$ S. Greene, ${ }^{177}$ A. Gurrola, ${ }^{177}$ R. Janjam, ${ }^{177}$ W. Johns, ${ }^{177}$ C. Maguire, ${ }^{177}$ A. Melo, ${ }^{177}$ H. Ni, ${ }^{177}$ K. Padeken, ${ }^{177}$ J. D. Ruiz Alvarez, ${ }^{177}$ P. Sheldon, ${ }^{177}$ S. Tuo, ${ }^{177}$ J. Velkovska ${ }^{177}$ Q. Xu, ${ }^{177}$ M. W. Arenton, ${ }^{178}$ P. Barria, ${ }^{178}$ B. Cox, ${ }^{178}$ R. Hirosky, ${ }^{178}$ M. Joyce, ${ }^{178}$ A. Ledovskoy, ${ }^{178} \mathrm{H}$. Li, ${ }^{178}$ C. Neu, ${ }^{178}$

T. Sinthuprasith, ${ }^{178}$ Y. Wang, ${ }^{178}$ E. Wolfe, ${ }^{178}$ F. Xia, ${ }^{178}$ R. Harr, ${ }^{179}$ P. E. Karchin,${ }^{179}$ N. Poudyal, ${ }^{179}$ J. Sturdy, ${ }^{179}$ P. Thapa, ${ }^{179}$ S. Zaleski, ${ }^{179}$ M. Brodski, ${ }^{180}$ J. Buchanan, ${ }^{180}$ C. Caillol, ${ }^{180}$ D. Carlsmith ${ }^{180}$ S. Dasu, ${ }^{180}$ L. Dodd, ${ }^{180}$ S. Duric, ${ }^{180}$ B. Gomber, ${ }^{180}$ M. Grothe, ${ }^{180}$ M. Herndon, ${ }^{180}$ A. Hervé, ${ }^{180}$ U. Hussain, ${ }^{180}$ P. Klabbers, ${ }^{180}$ A. Lanaro, ${ }^{180}$ A. Levine, ${ }^{180}$ K. Long, ${ }^{180}$ R. Loveless, ${ }^{180}$ T. Ruggles, ${ }^{180}$ A. Savin, ${ }^{180}$ N. Smith, ${ }^{180}$ W. H. Smith, ${ }^{180}$ and N. Woods ${ }^{180}$

(CMS Collaboration)

\author{
${ }^{1}$ Yerevan Physics Institute, Yerevan, Armenia \\ ${ }^{2}$ Institut für Hochenergiephysik, Wien, Austria \\ ${ }^{3}$ Institute for Nuclear Problems, Minsk, Belarus \\ ${ }^{4}$ Universiteit Antwerpen, Antwerpen, Belgium \\ ${ }^{5}$ Vrije Universiteit Brussel, Brussel, Belgium \\ ${ }^{6}$ Université Libre de Bruxelles, Bruxelles, Belgium \\ ${ }^{7}$ Ghent University, Ghent, Belgium \\ ${ }^{8}$ Université Catholique de Louvain, Louvain-la-Neuve, Belgium \\ ${ }^{9}$ Centro Brasileiro de Pesquisas Fisicas, Rio de Janeiro, Brazil \\ ${ }^{10}$ Universidade do Estado do Rio de Janeiro, Rio de Janeiro, Brazil \\ ${ }^{11 a}$ Universidade Estadual Paulista, São Paulo, Brazil \\ ${ }^{11 \mathrm{~b}}$ Universidade Federal do ABC, São Paulo, Brazil \\ ${ }^{12}$ Institute for Nuclear Research and Nuclear Energy, Bulgarian Academy of Sciences, Sofia, Bulgaria \\ ${ }^{13}$ University of Sofia, Sofia, Bulgaria \\ ${ }^{14}$ Beihang University, Beijing, China \\ ${ }^{15}$ Institute of High Energy Physics, Beijing, China \\ ${ }^{16}$ State Key Laboratory of Nuclear Physics and Technology, Peking University, Beijing, China \\ ${ }^{17}$ Tsinghua University, Beijing, China \\ ${ }^{18}$ Universidad de Los Andes, Bogota, Colombia \\ ${ }^{19}$ University of Split, Faculty of Electrical Engineering, Mechanical Engineering and Naval Architecture, Split, Croatia \\ ${ }^{20}$ University of Split, Faculty of Science, Split, Croatia \\ ${ }^{21}$ Institute Rudjer Boskovic, Zagreb, Croatia \\ ${ }^{22}$ University of Cyprus, Nicosia, Cyprus \\ ${ }^{23}$ Charles University, Prague, Czech Republic \\ ${ }^{24}$ Escuela Politecnica Nacional, Quito, Ecuador \\ ${ }^{25}$ Universidad San Francisco de Quito, Quito, Ecuador \\ ${ }^{26}$ Academy of Scientific Research and Technology of the Arab Republic of Egypt, Egyptian Network of High Energy Physics, \\ Cairo, Egypt \\ ${ }^{27}$ National Institute of Chemical Physics and Biophysics, Tallinn, Estonia
}


${ }^{28}$ Department of Physics, University of Helsinki, Helsinki, Finland

${ }^{29}$ Helsinki Institute of Physics, Helsinki, Finland

${ }^{30}$ Lappeenranta University of Technology, Lappeenranta, Finland

${ }^{31}$ IRFU, CEA, Université Paris-Saclay, Gif-sur-Yvette, France

${ }^{32}$ Laboratoire Leprince-Ringuet, Ecole polytechnique, CNRS/IN2P3, Université Paris-Saclay, Palaiseau, France

${ }^{33}$ Université de Strasbourg, CNRS, IPHC UMR 7178, Strasbourg, France

${ }^{34}$ Centre de Calcul de l'Institut National de Physique Nucleaire et de Physique des Particules, CNRS/IN2P3, Villeurbanne, France

${ }^{35}$ Université de Lyon, Université Claude Bernard Lyon 1, CNRS-IN2P3, Institut de Physique Nucléaire de Lyon, Villeurbanne, France

${ }^{36}$ Georgian Technical University, Tbilisi, Georgia

${ }^{37}$ Tbilisi State University, Tbilisi, Georgia

${ }^{38}$ RWTH Aachen University, I. Physikalisches Institut, Aachen, Germany

${ }^{39}$ RWTH Aachen University, III. Physikalisches Institut A, Aachen, Germany

${ }^{40}$ RWTH Aachen University, III. Physikalisches Institut B, Aachen, Germany

${ }^{41}$ Deutsches Elektronen-Synchrotron, Hamburg, Germany

${ }^{42}$ University of Hamburg, Hamburg, Germany

${ }^{43}$ Karlsruher Institut fuer Technology, Karlsrhue, Germany

${ }^{44}$ Institute of Nuclear and Particle Physics (INPP), NCSR Demokritos, Aghia Paraskevi, Greece

${ }^{45}$ National and Kapodistrian University of Athens, Athens, Greece

${ }^{46}$ National Technical University of Athens, Athens, Greece

${ }^{47}$ University of Ioánnina, Ioánnina, Greece

${ }^{48}$ MTA-ELTE Lendület CMS Particle and Nuclear Physics Group, Eötvös Loránd University, Budapest, Hungary

${ }^{49}$ Wigner Research Centre for Physics, Budapest, Hungary

${ }^{50}$ Institute of Nuclear Research ATOMKI, Debrecen, Hungary

${ }^{51}$ Institute of Physics, University of Debrecen, Debrecen, Hungary

${ }^{52}$ Indian Institute of Science (IISc), Bangalore, India

${ }^{53}$ National Institute of Science Education and Research, HBNI, Bhubaneswar, India

${ }^{54}$ Panjab University, Chandigarh, India

${ }^{55}$ University of Delhi, Delhi, India

${ }^{56}$ Saha Institute of Nuclear Physics, HBNI, Kolkata,India

${ }^{57}$ Indian Institute of Technology Madras, Madras, India

${ }^{58}$ Bhabha Atomic Research Centre, Mumbai, India

${ }^{59}$ Tata Institute of Fundamental Research-A, Mumbai, India

${ }^{60}$ Tata Institute of Fundamental Research-B, Mumbai, India

${ }^{61}$ Indian Institute of Science Education and Research (IISER), Pune, India

${ }^{62}$ Institute for Research in Fundamental Sciences (IPM), Tehran, Iran

${ }^{63}$ University College Dublin, Dublin, Ireland

${ }^{64 a}$ INFN Sezione di Bari, Bari, Italy

${ }^{64 \mathrm{~b}}$ Università di Bari, Bari, Italy

${ }^{64 \mathrm{c}}$ Politecnico di Bari, Bari, Italy

${ }^{65 \mathrm{a} I N F N ~ S e z i o n e ~ d i ~ B o l o g n a, ~ B o l o g n a, ~ I t a l y ~}$

${ }^{65 \mathrm{~b}}$ Università di Bologna, Bologna, Italy

${ }^{66 a}$ INFN Sezione di Catania, Catania, Italy

${ }^{66 \mathrm{~b}}$ Università di Catania, Catania, Italy

${ }^{67 \mathrm{a}}$ INFN Sezione di Firenze, Firenze, Italy

${ }^{67 \mathrm{~b}}$ Università di Firenze, Firenze, Italy

${ }^{68}$ INFN Laboratori Nazionali di Frascati, Frascati, Italy

${ }^{69 a}$ INFN Sezione di Genova, Genova, Italy

${ }^{69 \mathrm{~b}}$ Università di Genova, Genova, Italy

${ }^{70 a}$ INFN Sezione di Milano-Bicocca, Milano, Italy

${ }^{70 \mathrm{~b}}$ Università di Milano-Bicocca, Milano, Italy

${ }^{71 \mathrm{a}}$ INFN Sezione di Napoli, Napoli, Italy

${ }^{71 \mathrm{~b}}$ Università di Napoli 'Federico II', Napoli, Italy

${ }^{71 c}$ Università della Basilicata, Potenza, Italy

${ }^{71 d}$ Università G. Marconi, Roma, Italy

${ }^{72 a}$ INFN Sezione di Padova, Padova, Italy

${ }^{72 \mathrm{~b}}$ Università di Padova, Padova, Italy

${ }^{72 \mathrm{c}}$ Università di Trento, Trento, Italy

${ }^{73 a}$ INFN Sezione di Pavia, Pavia, Italy 


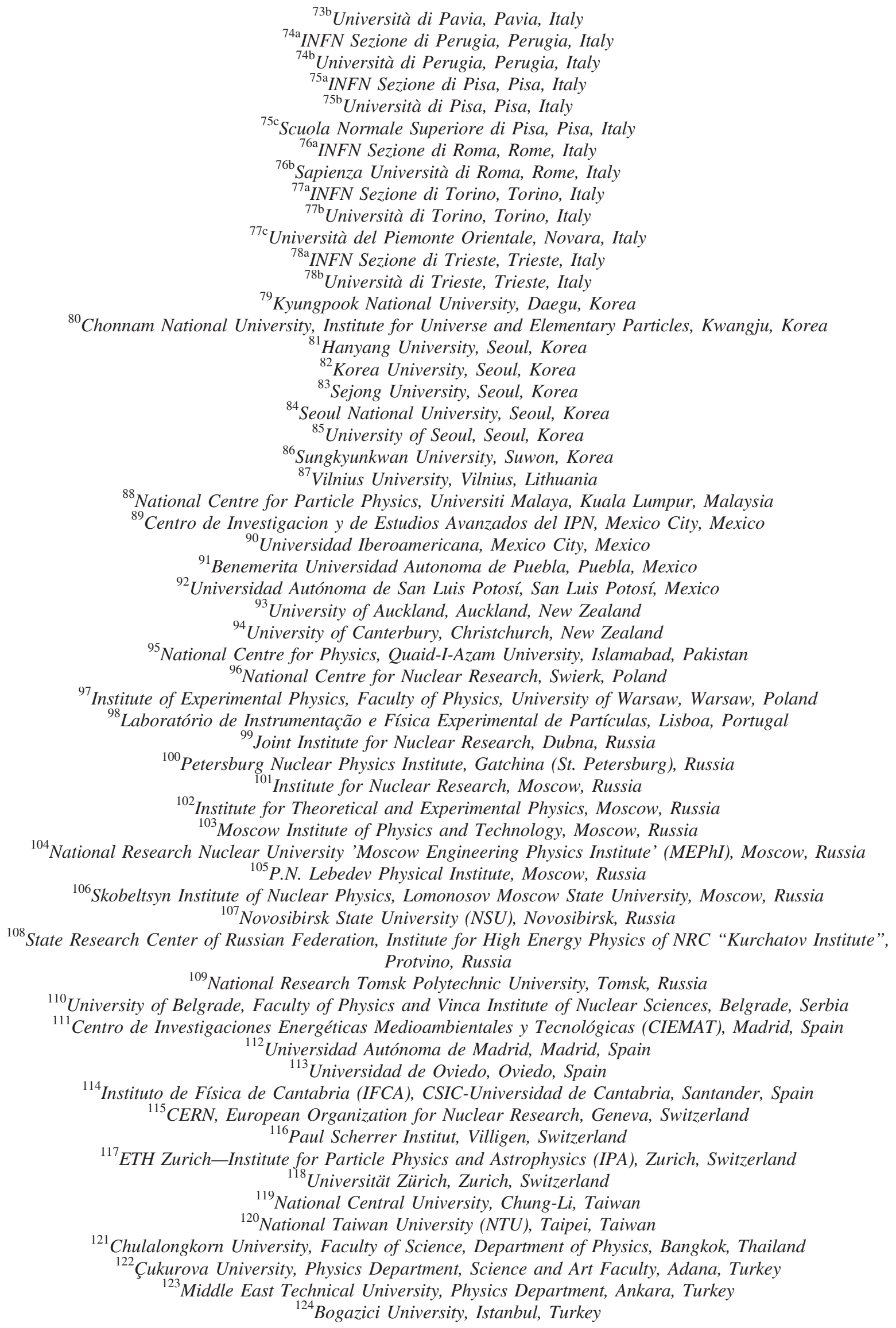


${ }^{125}$ Istanbul Technical University, Istanbul, Turkey

${ }^{126}$ Institute for Scintillation Materials of National Academy of Science of Ukraine, Kharkov, Ukraine

${ }^{127}$ National Scientific Center, Kharkov Institute of Physics and Technology, Kharkov, Ukraine

${ }^{128}$ University of Bristol, Bristol, United Kingdom

${ }^{129}$ Rutherford Appleton Laboratory, Didcot, United Kingdom

${ }^{130}$ Imperial College, London, United Kingdom

${ }^{131}$ Brunel University, Uxbridge, United Kingdom

${ }^{132}$ Baylor University, Waco, Texas, USA

${ }^{133}$ Catholic University of America, Washington, DC, USA

${ }^{134}$ The University of Alabama, Tuscaloosa, Alabama, USA

${ }^{135}$ Boston University, Boston, Massachusetts, USA

${ }^{136}$ Brown University, Providence, Rhode Island, USA

${ }^{137}$ University of California, Davis, Davis, California, USA

${ }^{138}$ University of California, Los Angeles, California, USA

${ }^{139}$ University of California, Riverside, Riverside, California, USA

${ }^{140}$ University of California, San Diego, La Jolla, California, USA

${ }^{141}$ University of California, Santa Barbara-Department of Physics, Santa Barbara, California, USA

${ }^{142}$ California Institute of Technology, Pasadena, California, USA

${ }^{143}$ Carnegie Mellon University, Pittsburgh, Pennsylvania, USA

${ }^{144}$ University of Colorado Boulder, Boulder, Colorado, USA

${ }^{145}$ Cornell University, Ithaca, New York, USA

${ }^{146}$ Fermi National Accelerator Laboratory, Batavia, Illinois, USA

${ }^{147}$ University of Florida, Gainesville, Florida, USA

${ }^{148}$ Florida International University, Miami, Florida, USA

${ }^{149}$ Florida State University, Tallahassee, Florida, USA

${ }^{150}$ Florida Institute of Technology, Melbourne, Florida, USA

${ }^{151}$ University of Illinois at Chicago (UIC), Chicago, Illinois, USA

${ }^{152}$ The University of Iowa, Iowa City, Iowa, USA

${ }^{153}$ Johns Hopkins University, Baltimore, Maryland, USA

${ }^{154}$ The University of Kansas, Lawrence, Kansas, USA

${ }^{155}$ Kansas State University, Manhattan, Kansas, USA

${ }^{156}$ Lawrence Livermore National Laboratory, Livermore, California, USA

${ }^{157}$ University of Maryland, College Park, Maryland, USA

${ }^{158}$ Massachusetts Institute of Technology, Cambridge, Massachusetts, USA

${ }^{159}$ University of Minnesota, Minneapolis, Minnesota, USA

${ }^{160}$ University of Mississippi, Oxford, Mississippi, USA

${ }^{161}$ University of Nebraska-Lincoln, Lincoln, Nebraska, USA

${ }^{162}$ State University of New York at Buffalo, Buffalo, New York, USA

${ }^{163}$ Northeastern University, Boston, Massachusetts, USA

${ }^{164}$ Northwestern University, Evanston, Illinois, USA

${ }^{165}$ University of Notre Dame, Notre Dame, Indiana, USA

${ }^{166}$ The Ohio State University, Columbus, Ohio, USA

${ }^{167}$ Princeton University, Princeton, New Jersey, USA

${ }^{168}$ University of Puerto Rico, Mayaguez, Puerto Rico

${ }^{169}$ Purdue University, West Lafayette, Indiana, USA

${ }^{170}$ Purdue University Northwest, Hammond, Indiana, USA

${ }^{171}$ Rice University, Houston, Texas, USA

${ }^{172}$ University of Rochester, Rochester, New York, USA

${ }^{173}$ Rutgers, The State University of New Jersey, Piscataway, New Jersey, USA

${ }^{174}$ University of Tennessee, Knoxville, Tennessee, USA

${ }^{175}$ Texas A\&M University, College Station, Texas, USA

${ }^{176}$ Texas Tech University, Lubbock, Texas, USA

${ }^{177}$ Vanderbilt University, Nashville, Tennessee, USA

${ }^{178}$ University of Virginia, Charlottesville, Virginia, USA

${ }^{179}$ Wayne State University, Detroit, Michigan, USA

${ }^{180}$ University of Wisconsin-Madison, Madison, Wisconsin, USA

${ }^{\mathrm{a}}$ Deceased.

${ }^{\mathrm{b}}$ Also at Vienna University of Technology, Vienna, Austria.

${ }^{c}$ Also at IRFU, CEA, Université Paris-Saclay, Gif-sur-Yvette, France. 
${ }^{\mathrm{d}}$ Also at Universidade Estadual de Campinas, Campinas, Brazil.

${ }^{\mathrm{e}}$ Also at Federal University of Rio Grande do Sul, Porto Alegre, Brazil.

${ }^{\mathrm{f}}$ Also at Université Libre de Bruxelles, Bruxelles, Belgium.

${ }^{g}$ Also at Institute for Theoretical and Experimental Physics, Moscow, Russia.

${ }^{\mathrm{h}}$ Also at Joint Institute for Nuclear Research, Dubna, Russia.

${ }^{\mathrm{i}}$ Also at Cairo University, Cairo, Egypt.

${ }^{\mathrm{j}}$ Also at Helwan University, Cairo, Egypt.

${ }^{\mathrm{k}}$ Also at Zewail City of Science and Technology, Zewail, Egypt.

${ }^{1}$ Also at Department of Physics, King Abdulaziz University, Jeddah, Saudi Arabia.

${ }^{\mathrm{m}}$ Also at Université de Haute Alsace, Mulhouse, France.

${ }^{\mathrm{n}}$ Also at Skobeltsyn Institute of Nuclear Physics, Lomonosov Moscow State University, Moscow, Russia.

${ }^{\circ}$ Also at CERN, European Organization for Nuclear Research, Geneva, Switzerland.

${ }^{\mathrm{p}}$ Also at RWTH Aachen University, III. Physikalisches Institut A, Aachen, Germany.

${ }^{\mathrm{q}}$ Also at University of Hamburg, Hamburg, Germany.

${ }^{\mathrm{r}}$ Also at Brandenburg University of Technology, Cottbus, Germany.

${ }^{\mathrm{s}}$ Also at Institute of Nuclear Research ATOMKI, Debrecen, Hungary.

${ }^{t}$ Also at MTA-ELTE Lendület CMS Particle and Nuclear Physics Group, Eötvös Loránd University, Budapest, Hungary.

${ }^{u}$ Also at Institute of Physics, University of Debrecen, Debrecen, Hungary.

${ }^{v}$ Also at IIT Bhubaneswar, Bhubaneswar, India.

${ }^{\mathrm{w}}$ Also at Institute of Physics, Bhubaneswar, India.

${ }^{\mathrm{x}}$ Also at Shoolini University, Solan, India.

${ }^{\mathrm{y}}$ Also at University of Visva-Bharati, Santiniketan, India.

${ }^{z}$ Also at Isfahan University of Technology, Isfahan, Iran.

${ }^{a a}$ Also at Plasma Physics Research Center, Science and Research Branch, Islamic Azad University, Tehran, Iran.

${ }^{\text {bb }}$ Also at Università degli Studi di Siena, Siena, Italy.

${ }^{c c}$ Also at International Islamic University of Malaysia, Kuala Lumpur, Malaysia.

${ }^{\mathrm{dd}}$ Also at Malaysian Nuclear Agency, MOSTI, Kajang, Malaysia.

${ }^{e e}$ Also at Consejo Nacional de Ciencia y Tecnología, Mexico city, Mexico.

${ }^{\mathrm{ff}}$ Also at Warsaw University of Technology, Institute of Electronic Systems, Warsaw, Poland.

${ }^{\mathrm{gg}}$ Also at Institute for Nuclear Research, Moscow, Russia.

${ }^{\text {hh }}$ Also at National Research Nuclear University 'Moscow Engineering Physics Institute' (MEPhI), Moscow, Russia.

${ }^{\text {ii }}$ Also at St. Petersburg State Polytechnical University, St. Petersburg, Russia.

${ }^{\mathrm{ij}}$ Also at University of Florida, Gainesville, Florida, USA.

${ }^{\mathrm{kk}}$ Also at P.N. Lebedev Physical Institute, Moscow, Russia.

${ }^{11}$ Also at California Institute of Technology, Pasadena, California, USA.

${ }^{\mathrm{mm}}$ Also at Budker Institute of Nuclear Physics, Novosibirsk, Russia.

${ }^{\mathrm{nn}}$ Also at Faculty of Physics, University of Belgrade, Belgrade, Serbia.

${ }^{\circ 0}$ Also at INFN Sezione di Pavia, Università di Pavia, Pavia, Italy.

${ }^{\mathrm{pp}}$ Also at University of Belgrade, Faculty of Physics and Vinca Institute of Nuclear Sciences, Belgrade, Serbia.

${ }^{\mathrm{qq}}$ Also at Scuola Normale e Sezione dell'INFN, Pisa, Italy.

${ }^{\mathrm{rr}}$ Also at National and Kapodistrian University of Athens, Athens, Greece.

${ }^{\text {ss }}$ Also at Riga Technical University, Riga, Finland.

"Also at Universität Zürich, Zurich, Switzerland.

${ }^{\text {uu }}$ Also at Stefan Meyer Institute for Subatomic Physics.

${ }^{\mathrm{vv}}$ Also at Adiyaman University, Adiyaman, Turkey.

${ }^{w w}$ Also at Istanbul Aydin University, Istanbul, Turkey.

${ }^{\mathrm{xx}}$ Also at Mersin University, Mersin, Turkey.

${ }^{\text {yy }}$ Also at Piri Reis University, Istanbul, Turkey.

${ }^{\mathrm{zz}}$ Also at Gaziosmanpasa University, Tokat, Turkey.

${ }^{\text {aaa }}$ Also at Ozyegin University, Istanbul, Turkey.

${ }^{\mathrm{bbb}}$ Also at Izmir Institute of Technology, Izmir, Turkey.

${ }^{c c c}$ Also at Marmara University, Istanbul, Turkey.

${ }^{\text {ddd }}$ Also at Kafkas University, Kars, Turkey.

${ }^{e e e}$ Also at Istanbul University, Faculty of Science, Istanbul, Turkey.

${ }^{\text {fff }}$ Also at Istanbul Bilgi University, Istanbul, Turkey.

${ }^{\text {ggg }}$ Also at Hacettepe University, Ankara, Turkey.

hhh Also at Rutherford Appleton Laboratory, Didcot, United Kingdom.

iiii Also at School of Physics and Astronomy, University of Southampton, Southampton, United Kingdom.

ijj Also at Monash University, Faculty of Science, Clayton, Australia.

${ }^{k k k}$ Also at Bethel University, St. Paul, Minnesota, USA. 


\footnotetext{
${ }^{\text {III }}$ Also at Utah Valley University, Orem, Utah, USA.

${ }^{\mathrm{mmm}}$ Also at Purdue University, West Lafayette, Indiana, USA.

${ }^{n n n}$ Also at Beykent University, Istanbul, Turkey.

${ }^{000}$ Also at Bingol University, Bingol, Turkey.

${ }^{\mathrm{ppp}}$ Also at Sinop University, Sinop, Turkey.

${ }^{\mathrm{qqq}}$ Also at Mimar Sinan University, Istanbul, Istanbul, Turkey.

${ }^{\mathrm{rrr}}$ Also at Texas A\&M University at Qatar, Doha, Qatar.

${ }^{\text {sss }}$ Also at Kyungpook National University, Daegu, Korea.
} 